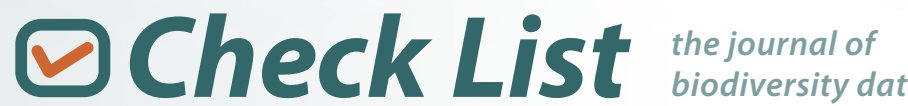

\section{Angiosperm diversity of the Great Indian Bustard Wildlife Sanctuary: a semi-arid grassland, Maharashtra, India}

\author{
Jayanthi Janakiraman* and Jeewan Singh Jalal \\ Botanical Survey of India, Western Regional Centre, 7 Koregaon Road, Pune - 411001, Maharashtra, India \\ * Corresponding author. E-mail: jayanthi.bsi@gmail.com
}

\begin{abstract}
The Great Indian Bustard Wildlife sanctuary is a semi-arid grassland ecosystem spread over an area of $1,222 \mathrm{~km}^{2}$ in the Solapur and Ahmednagar districts of Maharashtra, India. It is an abode of the critically endangered bird, the Great Indian Bustard. A total of 436 plants belonging to 259 genera and 67 families are reported in the present study from the sanctuary, including 22 endemic taxa. Grasses form one of the dominant components of the ecosystem and are represented by about 67 species. The sanctuary is facing severe habitat loss and degradation, posing a threat to its biodiversity. This paper provides a comprehensive documentation of the floristic diversity of the sanctuary. Threats and conservation measures are also discussed.
\end{abstract}

Key words: inventory, floristics, Nannaj, protected area, Solapur.

\section{INTRODUCTION}

Grasslands are a major component of the world's vegetation, covering about $24 \%$ (Shantz 1954). They are considered to be a major potential sink for carbon, as grassland soil stores more carbon compared to forest soil (Dinakaran et al. 2011). Grasslands occur in places where temperature, precipitation and physical factors such as soil and drainage combine to create conditions that are too dry for trees to grow. Grasslands have evolved through grazing, drought and periodic fire, and therefore almost all grasslands are maintained by either one or a combination of these factors. They provide habitats for many different animals, birds, insects and lizards, which depend on each other for survival (Allaby 2006; Nancy et al. 2013).

Despite their widespread occurrence, grasslands are shrinking at an alarming rate globally, and are fast becoming one of the most endangered of ecosystems (IUCN 2014). The rapid decline of natural grasslands is due to several reasons. Traditionally, grasslands and scrub have been considered as "wasteland". They are prime targets for development projects. About $16 \%$ of tropical grasslands have been converted for agriculture or urban development. In forming relatively open areas with low-stature vegetation which intercepts high light intensity, grasslands are also vulnerable to invasive species (Wagner 1989). Their structure and function make them one of the most vulnerable of terrestrial ecosystems to global climate change (Schlesinger 1997; Mooney and Hobbs 2000; Lejeune and Seastedt 2001). Grassland studies are therefore of paramount importance and a pre-requisite for conservation and management of wildlife (Rodgers and Sawarkar 1988; Rahmani 1992).

India has an estimated coverage of grasslands and shrub lands that vary from 3.7 percent to as much as 12 percent of the total area (UNDP 2012). Whyte (1957) has classified Indian grasslands into eight types while Champion and Seth (1968) recognized only three broad categories. The Indian Council of Agricultural Research (ICAR) classified them into five categories, namely Sehima-Dichanthium type, Dichanthium-Cenchrus-Lasiurus type, Phragmites-Saccharum-Imperata type, ThemedaArundinella type and temperate and alpine cover, based on the dominant grass composition (Dabadghao and Shankarnarayan 1973). Based on eco-climatic factors, the Indian grasslands fall under four categories: Himalayan pastures, Terai grasslands, semi-arid grasslands and Shola grasslands. Among these, semi-arid grasslands are found in Western India, Central India and Deccan and are characterized by grassland tracts with patches of thorn forests.

The Bustard Sanctuary comes under the semi-arid grasslands category and is the abode of the Critically Endangered species the Great Indian Bustard [Ardeotis nigriceps (Vigors, 1831)] (Birdlife International 2011). This ostrich-like bird occurs in the semi-arid grasslands and deserts of India. It prefers open landscapes, and in particular grassy plains in arid and semi-arid areas interspersed with scrub and low bushes (Rahmani 2006; 
Munjpara et al. 2012). It is locally known as "Maldhok" in Marathi. Floristic studies in the semi-arid part of Deccan are few when compared to the Western Ghats region of Maharashtra. However, some publications have described areas adjacent to the Bustard Sanctuary (Naik 1979; Pradhan and Singh 1999; Suryavanshi and Bachulkar 2011; Das Das and Singh 2011; Gaikwad et al. 2012; Das Das 2012). No floristic documentation of the Bustard Sanctuary is available to-date, and hence, this study was undertaken with the primary objective of the Botanical Survey of India to explore, inventory and document plant diversity in protected areas of India.

\section{MATERIALS AND METHODS \\ Study area}

The Great Indian Bustard Wildlife Sanctuary (GIBWLS) was declared a sanctuary vide notification No. WLP-1078/72634/FI in 1979 by the Indian Board for Wildlife to protect the critically endangered and endemic bird, the Great Indian Bustard (GIB). It is located in Solapur and Ahmednagar district of Maharashtra and lies between $17^{\circ} 22^{\prime} 17^{\prime \prime}$ to $18^{\circ} 54^{\prime} 42^{\prime \prime} \mathrm{N}$ and $074^{\circ} 23^{\prime} 34^{\prime \prime}$ to $076^{\circ} 15^{\prime} \mathrm{O} 1^{\prime \prime}$ E (Figure 1). The sanctuary is spread over an area of $c a .1,222 \mathrm{~km}^{2}$. It also includes about 393 villages within its boundary. In general, the terrain of the sanctuary is flat and undulating with an average elevation of 500 to $600 \mathrm{~m}$ above mean sea level and covered by a thin mantle of black cotton soil. Apart from black soil, the soil colour varies from reddish brown to coarse grey. The study area comes under the forest types of semi-arid biotope, open scrublands and southern tropical thorn forest (Champion and Seth 1968). The common tree species in this ecosystem are: Acacia nilotica, A. catechu, Capparis decidua, Senna auriculata, Mimosa hamata and Ziziphus mauritiana. The common herbs include Aeschynomene indica, Alysicarpus vaginalis, Biophytum sensitivum, Cleome viscosa, Glossocardia bosvallia, Indigofera cordifolia and Indigofera linifolia. The common grasses are Apluda mutica, Chrysopogon fulvus, Cymbopogon martini, Dichanthium annulatum, Heteropogon contortus, Ischaemum afrum and Setaria pumila. Factors such as poor soil quality, frequent fire and erosion contribute to the lack of regeneration of the main species and deterioration of native vegetation. The area belongs to the rain shadow region and is prone to annual droughts. June to September is the southwest monsoon season. October and November constitute the post-monsoon or retreating monsoon season. The average annual rainfall in the district is between $550 \mathrm{~mm}$ to $650 \mathrm{~mm}$. May is the hottest month with mean daily maximum temperature of $39.9^{\circ} \mathrm{C}$ and the mean daily minimum of $25.1^{\circ} \mathrm{C}$. Grasses form one of the dominant components of the ecosystem. Agriculture is the main occupation of the people in this region. The main crops cultivated include rice, jowar, bajra, pulses, cotton, sugarcane, groundnut,

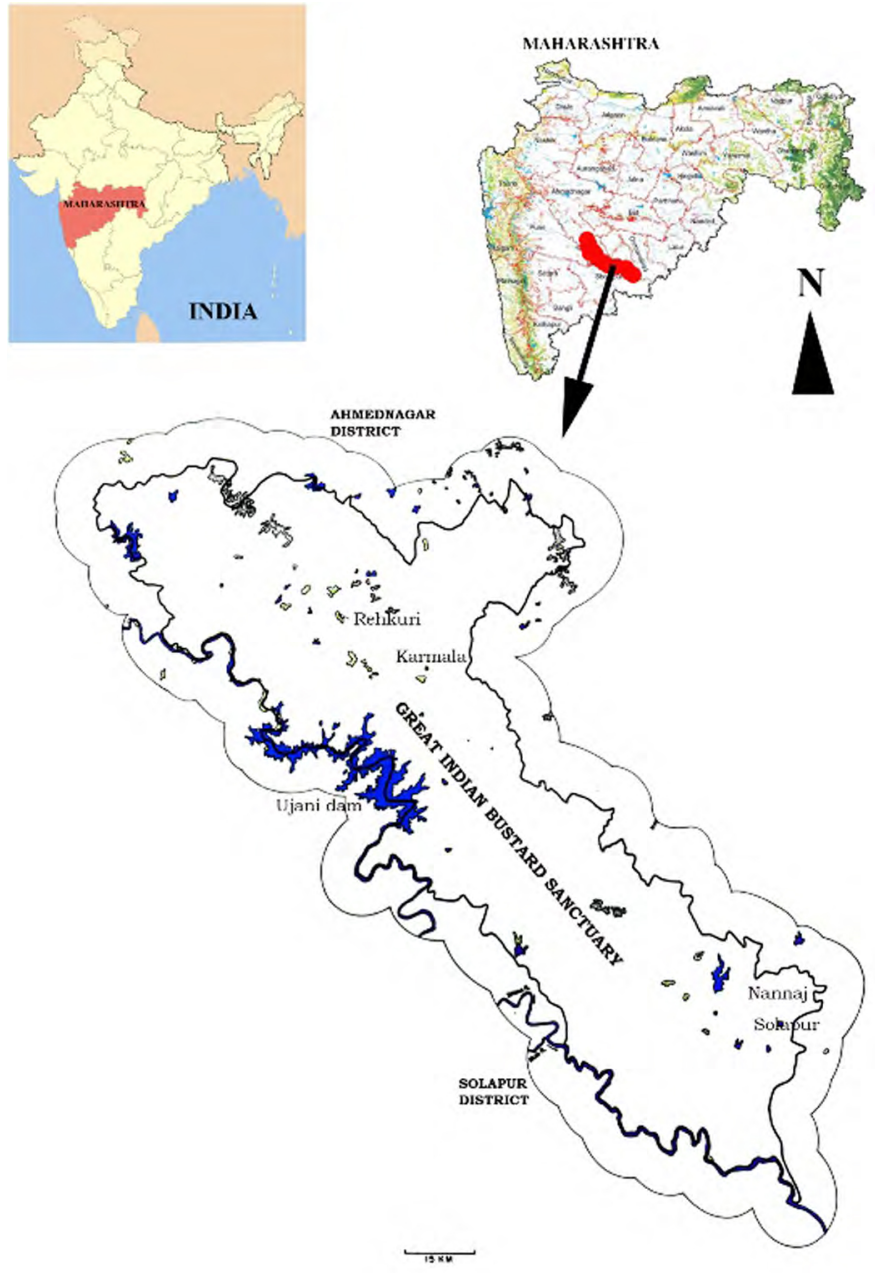

Figure 1. Map showing the study area.

sunflower, turmeric, onion and vegetables such as lady's finger, brinjal, gourds and spinach. Fruits such as grapes and bananas are also cultivated. Plants such as Parkinsonia aculeata, Eucalyptus tereticornis, Acacia auriculiformis, Dodonaea viscosa, Albizia lebbeck, Casuarina equisetifolia, Duranta repens, Gliricidia sepium, Azadirachta indica, etc., are planted near the forest guest houses and along the roadsides.

\section{Data collection}

Regular plant exploration tours were undertaken from July 2010 to December 2012. Field trips were organized in different seasons and covered all parts of the study area. In each field trip, the data collected include: habit, habitat, flowering period and local names. Plant samples of all the species were collected from different areas of the sanctuary. The specimens were critically studied for morphological details and for correct identification. Identification was confirmed by consulting standard literature (Cooke 1901; 1908; Naik 1979; Sharma et al. 1996; Pradhan and Singh 1999; Singh and Karthikeyan 2000; Singh et al. 2001; Bhagat et al. 2008; Karthikeyan et al. 2009) and through reference against identified herbarium specimens at Botanical Survey of India, Western 
Regional Centre herbarium, Pune (BSI) and Blatter herbarium, Mumbai (BLAT). Web databases such as The Plant List (2013), International Plant Names Index (IPNI 2013), Tropicos, Biodiversity Heritage Library (BHL 2013), Global Biodiversity Information Facility (GBIF 2013) and Kew World Checklist of Selected Plant Families (WCSP 2013) were also consulted for correct nomenclature, author citation and additional information. Author names have been standardised based on Brummitt and Powell (2004). The voucher specimens are deposited at BSI Herbarium Pune, Maharashtra.

\section{RESULTS}

Intensive study on the floristic diversity of the Great Indian Bustard Sanctuary resulted in documentation of a total of 436 taxa (Table 1). They belong to 259 genera spread over 67 families. Of these, 317 (73\%) taxa belong to dicotyledons and 119 (27\%) taxa to monocotyledons (Figure 2). The most dominant families are Poaceae (67 taxa), Fabaceae (41 taxa), Cyperaceae (30 taxa), Asteraceae (29 taxa) and Euphorbiaceae (24 taxa) (Figure 3). Genera such as Cyperus, Euphorbia, Ipomoea, Senna, Acacia and Crotalaria are dominant, possessing greatest species diversity (Figure 4). The flora of the sanctuary is categorized into different life-forms such as trees, shrubs, herbs and climbers in which herbs dominate the flora followed by shrubs and climbers (Figure 5). Trees are poorly represented with only 30 taxa, most of which belong to the genus Acacia which is

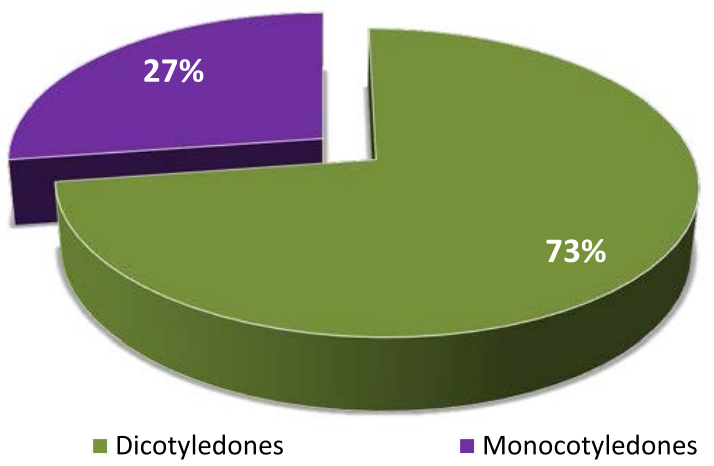

Figure 2. Distribution of dicots and monocots.

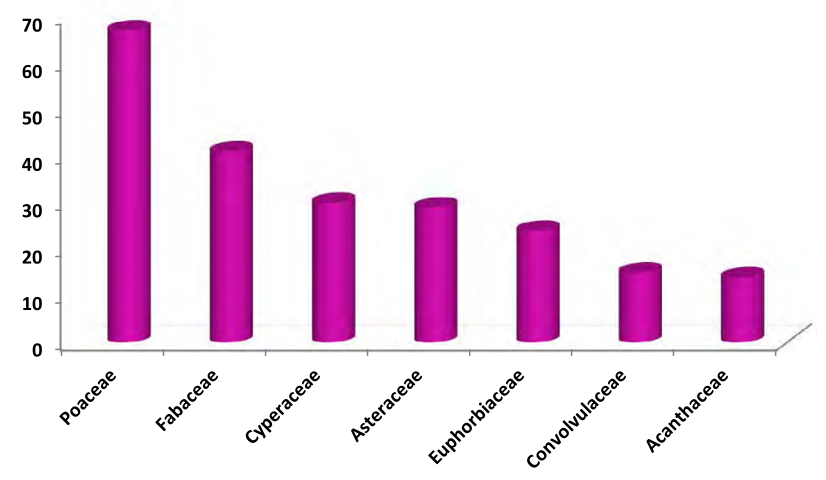

Figure 3. Dominant families of GIBWLS.

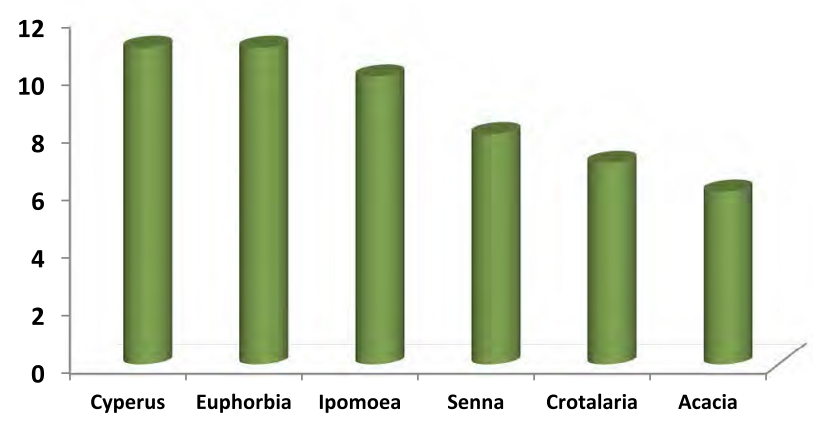

Figure 4. Dominant genera in GIBWLS.

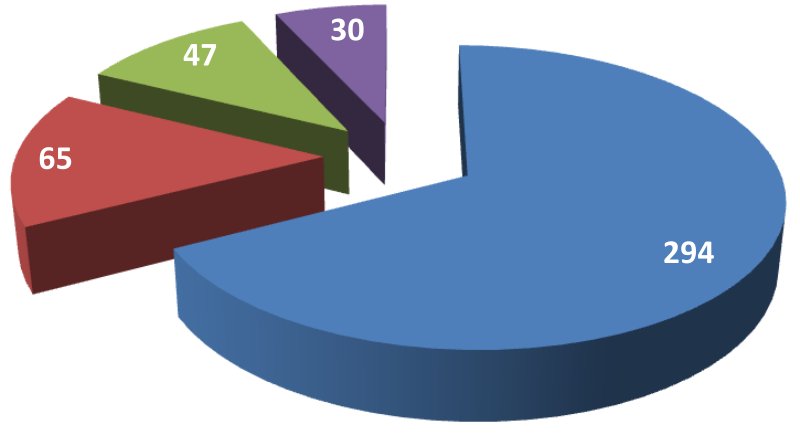

Herbs Shrubs Climbers Trees

Figure 5. Distribution of life-forms in GIBWLS.

characteristic of the semi-arid ecosystem. Twenty-two endemic taxa were also recorded during the present study: Cleome simplicifolia, Crotalaria filipes, Crotalaria vestita, Vigna indica, Hardwickia binata, Dichrostachys cinerea var. indica, Neonotis montholoni, Glossocardia bosvallia, Tricholepis radicans, Exacum pumilum, Hemigraphis urens, Euphorbia notoptera, Aristida stocksii, Isachne borii, Iseilema anthephoroides, Lophopogon tridentatus, Oropetium roxburghianum, Oropetium villosulum, Sehima sulcatum, Spodiopogon rhizophorus, Tragus mongolorum and Tripogon jacquemontii.

\section{DISCUSSION}

In India, less than one percent of grasslands come under the protected area network, of which $0.24 \%$ lies in the semi-arid zone. Some of the protected areas of arid and semi-arid grasslands serve as an important genetic resource in the form of grass and shrub species which are vital for maintaining ecological balance and for food security of the country. These protected areas therefore should not only be considered as key for wildlife conservation, but also as gene banks given that modern cereals have originated from wild grasses (Government of India 2006).

The GIBWLS, which is a grassland ecosystem that accounts for 67 wild grass species and 41 wild legumes, is facing rapid industrialization, conversion of grassland to farmlands and an increase in human population 
during the last 30 years. There are also several microindustries, stone and brick quarries and sugar cane factories located or emerging around the sanctuary's periphery. The cumulative impact of all these land-use pressures has lead to severe degradation of habitat, which in turn is leading to a decline in the population of Great Indian Bustard (Dutta et al. 2011). The remaining suitable habitat consists of small and scattered grassland patches protected under the Drought Prone Areas Programme plots, which was introduced by the Central Government. The total aggregate area of these scattered patches is not more than $400 \mathrm{~km}^{2}$, with the biggest patch located in the village called Nannaj that lies to the north of Solapur town.

Plantations of trees such as Gliricidia, Azadirachta, Eucalyptus and Acacia auriculiformis in the grasslands will adversely affect the habitat's suitability for birds and animals and hence they should be removed. The spread of obnoxious invasive weeds such as Hyptis suaveolens, Parthenium hysterophorus, Lantana camara and Cassia uniflora should be controlled and they should be removed from the grassland. Prioritising conservation measures at potential areas such as Nannaj, Mardi, Akolekati, Karamba, Gangevadi, Chapadgaon, Rehkuri and Korti is essential, as these areas harbour several endemic species as well as forming habitat for Ceropegia bulbosa; a relatively rare species in the sanctuary.

A total of 22 peninsular endemic species were collected and recorded from these areas during this study. It is observed that 24grass species havepotentialfoddervalue:
Apluda mutica, Bothriochloa pertusa, Cenchrus setigerus, Chloris gayana, Cynodon dactylon, Dactyloctenium aegyptiacum, Dichanthium foveolatum, Digitaria ternata, Echinochloa colonum, Eleusine indica, Eragrostis pilosa, Eragrostis tenella, Eragrostis viscosa, Heteropogon contortus, Melanocenchris jacquemontii, Oryza sativa, Panicum hippothrix, Panicum curviflorum, Paspalidium flavidum, Pennisetum pedicellatum, Pennisetum purpureum, Sehima ischaemoides, Sporobolus indicus and Tetrapogon tenellus. These grasses may be promoted in the sanctuary. Flowers of species such as Indigofera linifolia, Alysicarpus spp., Cyanotis fasciculata and Chlorophytum tuberosum, etc. are the main attraction for many beetles and insects. During the study, it was also observed that species such as Apluda mutica, Aristida redacta, A. stocksii, Echinochloa colona, Iseilema anthephoroides, Lophopogon tridentatus, Melanocenchris jacquemontii, Oropetium spp., Ledebouria hyacinthina, Chlorophytum tuberosum, Boerhavia diffusa, Boerhavia erecta, Vigna indica, Lepidagathis cristata, Spermacoce pusilla, Trichodesma indicum, Cyanotis fasciculata, Ipomoea eriocarpa, Indigofera cordifolia, Lavandula bipinnata, etc. support a variety of insects, beetles, and grasshoppers which are the main diet of many grassland birds and animals. Care must be provided to help increase the population of these plant species.

Furthermore, GIS studies should be undertaken for mapping the area to identify the potential grassland patches and level of disturbances in the landscape so as to plan for suitable habitat restoration programmes. Considering this scenario of habitat loss and degradation,

Table 1. List of species in the study area (abbreviations: $\mathrm{H}=$ herbs, $\mathrm{S}=$ shrubs, $\mathrm{C}=$ climbers, $\mathrm{T}=$ trees, ${ }^{*}=$ species added based on literature as well as observation in field in vegetative condition.

\begin{tabular}{|c|c|c|c|}
\hline Taxa & Habit & Flowers & Field No. \\
\hline \multicolumn{4}{|l|}{ Acanthaceae } \\
\hline Andrographis echioides (L.) Nees & $\mathrm{H}$ & August - September & 198125 \\
\hline Barleria prionitis L. & $\mathrm{S}$ & November - December & J J J 199362 \\
\hline Dicliptera paniculata (Forssk.) I. Darbysh. & $\mathrm{H}$ & October - December & J J 199311 \\
\hline Dicliptera verticillata (Forssk.) C.Chr. & $\mathrm{H}$ & November - December & 199323 \\
\hline Hemigraphis urens (Roth) J.R.I. Wood & $\mathrm{H}$ & November - December & 199389 \\
\hline Hygrophila schulli (Buch.-Ham.) M.R.Almeida \& S.M.Almeida & $\mathrm{H}$ & November - December & 199361 \\
\hline Justicia diffusa Willd. & $\mathrm{H}$ & August - May & * \\
\hline Justicia japonica Thunb. & $\mathrm{H}$ & August - September & 198002 \\
\hline Lepidagathis cristata Willd. & $\mathrm{H}$ & August - December & 198158 \\
\hline Lepidagathis trinervis Nees & $\mathrm{H}$ & November - May & * \\
\hline Ruellia patula Jacq. & $\mathrm{H}$ & July - August & J J 199257 \\
\hline Ruellia prostrata Poir. & $\mathrm{H}$ & July - August & 199405 \\
\hline Rungia elegans Dalzell \& A. Gibson & $\mathrm{H}$ & November - December & 199360 \\
\hline Rungia repens (L.) Nees & $\mathrm{H}$ & August - September & 198148 \\
\hline \multicolumn{4}{|l|}{ Agavaceae } \\
\hline Agave americana $\mathrm{L}$. & $\mathrm{H}$ & October - December & J J J 199491 \\
\hline \multicolumn{4}{|l|}{ Aizoaceae } \\
\hline Trianthema portulacastrum L. & $\mathrm{H}$ & September - October & 198277 \\
\hline \multicolumn{4}{|l|}{ Amaranthaceae } \\
\hline Achyranthes aspera $\mathrm{L}$. & $\mathrm{H}$ & October - December & J J 198052 \\
\hline Aerva javanica (Burm. f.) Juss. & $\mathrm{H}$ & October - December & 199467 \\
\hline Alternanthera pungens Kunth & $\mathrm{H}$ & September - December & 198222 \\
\hline
\end{tabular}


Table 1. Continued.

\begin{tabular}{|c|c|c|c|}
\hline Taxa & Habit & Flowers & Field No. \\
\hline Alternanthera sessilis (L.) R. Br. ex DC. & $\mathrm{H}$ & August - December & 198131 \\
\hline Alternanthera tenella Colla & $\mathrm{H}$ & August - December & 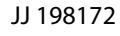 \\
\hline Amaranthus spinosus $\mathrm{L}$. & $\mathrm{H}$ & July - December & 199239 \\
\hline Amaranthus viridis L. & $\mathrm{H}$ & August - December & 198270 \\
\hline Celosia argentea L. & $\mathrm{H}$ & July - October & 198205 Jנ \\
\hline Digera muricata (L.) Mart. & $\mathrm{H}$ & August - October & J J 198255 \\
\hline Gomphrena serrata L. & $\mathrm{H}$ & August - September & 198054 \\
\hline Pupalia lappacea (L.) Juss. & $\mathrm{H}$ & August - September & J J 198254 \\
\hline \multicolumn{4}{|l|}{ Anacardiaceae } \\
\hline Rhus mysorensis G. Don & $\mathrm{s}$ & July - December & J J 199288 \\
\hline \multicolumn{4}{|l|}{ Apiaceae } \\
\hline Centella asiatica (L.) Urb. & $\mathrm{H}$ & September-October & J J 199412 \\
\hline \multicolumn{4}{|l|}{ Apocynaceae } \\
\hline Catharanthus pusillus (Murr.) G. Don & $\mathrm{H}$ & September-October & J J J 198193 \\
\hline Catharanthus roseus (L.) G. Don & $\mathrm{s}$ & September - December & 199477 \\
\hline \multicolumn{4}{|l|}{ Araceae } \\
\hline Colocasia esculenta (L.) Schott. & $\mathrm{H}$ & July - December & J J 199490 \\
\hline \multicolumn{4}{|l|}{ Arecaceae } \\
\hline Borassus flabellifer L. & $\mathrm{T}$ & March - June & * \\
\hline Phoenix sylvestris (L.) Roxb. & $\mathrm{T}$ & March - June & * \\
\hline \multicolumn{4}{|l|}{ Aristolochiaceae } \\
\hline Aristolochia bracteolata Lam. & $\mathrm{H}$ & July - September & 199254 \\
\hline \multicolumn{4}{|l|}{ Asclepiadaceae } \\
\hline Asclepias curassavica L. & $\mathrm{H}$ & October - February & 199471 \\
\hline Calotropis gigantea (L.) R. Br. & $\mathrm{s}$ & Almost throughout the year & 199235 \\
\hline Calotropis procera (Aiton.) R. Br. & $\mathrm{s}$ & Almost throughout the year & 198401 \\
\hline Caralluma adscendens (Roxb.) R. Br. & $\mathrm{H}$ & July - September & J J 198063 \\
\hline Ceropegia bulbosa Roxb. & c & August - September & J J 198119 \\
\hline Dregea volubilis (L.f.) Benth. ex Hook.f & c & August - October & 198141 \\
\hline Marsdenia sylvestris (Retz.) P.I. Forst. & c & July - October & J J 199258 \\
\hline Pentatropis capensis (L. f.) Bullock & c & July - October & J J 199283 \\
\hline Pergularia daemia (Forssk.) Choiv. & c & August - September & 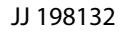 \\
\hline Secamone emetica (Retz.) R. Br. & c & July - August & J J 199255 \\
\hline Tylophora indica (Burm. f.) Merr. & C & August - September & J J 199478 \\
\hline \multicolumn{4}{|l|}{ Asteraceae } \\
\hline Acanthospermum hispidum DC. & $\mathrm{H}$ & September-October & J J J 198179 \\
\hline Ageratum conyzoides $\mathrm{L}$. & $\mathrm{H}$ & September - December & J J 194802 \\
\hline Anaphalis subdecurrens (DC.) Gamble & $\mathrm{H}$ & September - December & 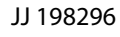 \\
\hline Bidens biternata (Lour.) Merr. \& Sherff. & $\mathrm{H}$ & September - December & J J 198098 \\
\hline Blainvillea acmella (L.) Philipson & $\mathrm{H}$ & September - December & J J 198134 \\
\hline Blumea axillaris (Lam.) DC. & $\mathrm{H}$ & October - December & 199320 \\
\hline Blumea lacera (Burm. f.) DC. & $\mathrm{H}$ & August - December & J J 199315 \\
\hline Blumea oxyodonta DC. & $\mathrm{H}$ & October - December & J J 199335 \\
\hline Caesulia axillaris Roxb., & $\mathrm{H}$ & September - October & 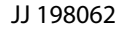 \\
\hline Cyanthillium cinereum (L.) H. Rob. & $\mathrm{H}$ & September-October & J J 198110 \\
\hline Cyathocline purpurea (Buch.-Ham. ex D. Don) O. Ktze. & $\mathrm{H}$ & October - December & J J J 199316 \\
\hline Dicoma tomentosa Cass. & $\mathrm{H}$ & October - December & J J 198088 \\
\hline Echinops echinatus Roxb. & $\mathrm{H}$ & October - December & J J 199327 \\
\hline Eclipta prostrata (L.) L. & $\mathrm{H}$ & August - October & 198221 \\
\hline Emilia sonchifolia (L.) DC. & $\mathrm{H}$ & September - October & J J 198109 \\
\hline Flaveria trinervia (Spreng.) C. Mohr. & $\mathrm{H}$ & July - August & J J 199297 \\
\hline Glossocardia bosvallia (L. f.) DC. & $\mathrm{H}$ & September - October & J J 198015 \\
\hline Goniocaulon indicum (Klein ex Willd.) Clarke & $\mathrm{H}$ & November - December & J J 199388 \\
\hline Grangea maderaspatana (L.) Poir. & $\mathrm{H}$ & July-August & 199223 \\
\hline Lagascea mollis Cav. & $\mathrm{H}$ & August - September & J J 199215 \\
\hline Launaea procumbens (Roxb.) Ramayya \& Rajagopal & $\mathrm{H}$ & August - September & J J 199295 \\
\hline Parthenium hysterophorus L. & $\mathrm{H}$ & August - September & J J J 198212 \\
\hline Pluchea lanceolata (DC.) C.B. Clarke & S & October - December & J J 199400 \\
\hline Pseudognaphalium luteo-album (L.) Hillard \& B.L. Burtt. & H & September - December & J J 199352 \\
\hline Pulicaria wightiana C. B. Clarke & $\mathrm{H}$ & August - September & J J 198021 \\
\hline
\end{tabular}


Table 1. Continued.

\begin{tabular}{|c|c|c|c|}
\hline Taxa & Habit & Flowers & Field No. \\
\hline Sphaeranthus indicus L. & $\mathrm{H}$ & November - December & 199371 \\
\hline Tricholepis radicans (Roxb.) DC. & $\mathrm{H}$ & September - October & 198051 \\
\hline Tridax procumbens $\mathrm{L}$. & $\mathrm{H}$ & September-October & 198020 \\
\hline Xanthium indicum Koen. & $\mathrm{s}$ & October - December & 198163 \\
\hline \multicolumn{4}{|l|}{ Bignoniaceae } \\
\hline Dolichandrone falcata (Wall. ex DC.) Seem. & $\mathrm{T}$ & August - September & 198171 \\
\hline \multicolumn{4}{|l|}{ Boraginaceae } \\
\hline Coldenia procumbens $\mathrm{L}$. & $\mathrm{H}$ & July - August & 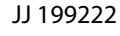 \\
\hline Cordia dichotoma Forst. f. & $\mathrm{T}$ & July - August & 199248 \\
\hline Cordia sinensis Lam. & $\mathrm{T}$ & July - August & J J 199287 \\
\hline Heliotropium bracteatum R. Br. & $\mathrm{H}$ & September-October & 198070 \\
\hline Heliotropium indicum L. & $\mathrm{H}$ & Frequently throughout the year & 199472 \\
\hline Heliotropium ovalifolium Forssk. & $\mathrm{H}$ & July - September & 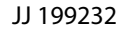 \\
\hline Heliotropium supinum L. & $\mathrm{H}$ & October - December & J J 199381 \\
\hline Trichodesma indicum (L.) Lehm. & $\mathrm{H}$ & August - September & J J 198091 \\
\hline Trichodesma zeylanicum (Burm. f.) R. Br. & $\mathrm{H}$ & August - September & J 198188 \\
\hline \multicolumn{4}{|l|}{ Brassicaceae } \\
\hline Brassica juncea (L.) Czern. & $\mathrm{H}$ & June - October & J J 199476 \\
\hline Thlaspi arvensis L. & $\mathrm{H}$ & July - August & J J 199298 \\
\hline \multicolumn{4}{|l|}{ Cactaceae } \\
\hline Opuntia elatior Mill. & $\mathrm{s}$ & August - September & * \\
\hline \multicolumn{4}{|l|}{ Caesalpiniaceae } \\
\hline Bauhinia racemosa Lam. & $\mathrm{T}$ & June - August & 198197 \\
\hline Caesalpinia bonduc (L.) Roxb. & s & July - December & J J 199236 \\
\hline Chamaecrista mimosoides (L.) Greene & $\mathrm{H}$ & September-October & 198049 \\
\hline Hardwickia binata Roxb., & $\mathrm{T}$ & August - December & 199246 \\
\hline Senna alata (L.) Roxb. & s & July - December & 199475 \\
\hline Senna auriculata (L.) Roxb. & s & Almost throughout the year & J 198031 \\
\hline Senna italica Mill. & $\mathrm{s}$ & July - August & 199281 \\
\hline Senna obtusifolia (L.) H.S. Irwin \& Barneby & $\mathrm{s}$ & September - October & J J 199402 \\
\hline Senna occidentalis (L.) Link & $S$ & August - December & J J 198113 \\
\hline Senna sophera (L.) Roxb. & S & July - August & J J 199289 \\
\hline Senna tora (L.) Roxb. & $\mathrm{s}$ & July-August & 199406 נJ \\
\hline Senna uniflora (Mill.) H.S. Irwin \& Barneby & $S$ & September - December & J 198030 \\
\hline \multicolumn{4}{|l|}{ Capparaceae } \\
\hline Capparis decidua (Forssk.) Edgew. & $\mathrm{T}$ & July - August & J J 199201 \\
\hline Capparis divaricata Lam. & $\mathrm{s}$ & July-December & 198196 נJ \\
\hline Capparis grandis L.f. & $\mathrm{T}$ & April-August & J J 199275 \\
\hline Cleome chelidonii L. f. & $\mathrm{H}$ & July - October & J J 199221 \\
\hline Cleome gynandra L. & $\mathrm{H}$ & July - October & 198263 \\
\hline Cleome simplicifolia (Cambess.) Hook. f. \& Thoms. & $\mathrm{H}$ & July - September & J J 198013 \\
\hline Cleome viscosa L. & $\mathrm{H}$ & July - September & J J 198286 \\
\hline \multicolumn{4}{|l|}{ Caryophyllaceae } \\
\hline Polycarpaea corymbosa (L.) Lam. & $\mathrm{H}$ & August - September & J J 198012 \\
\hline \multicolumn{4}{|l|}{ Casuarinaceae } \\
\hline Casuarina equisetifolia L. & $\mathrm{T}$ & August - October & J 199500 \\
\hline \multicolumn{4}{|l|}{ Colchicaceae } \\
\hline Iphigenia indica (L.) Kunth & $\mathrm{H}$ & July - September & J J 198147 \\
\hline \multicolumn{4}{|l|}{ Commelinaceae } \\
\hline Commelina benghalensis L. & $\mathrm{H}$ & July - September & J J 199489 \\
\hline Commelina diffusa Burm.f. & $\mathrm{H}$ & July - September & J J 198059 \\
\hline Commelina erecta $\mathrm{L}$. & $\mathrm{H}$ & July - September & J J 199284 \\
\hline Commelina forskalaei Vahl & $\mathrm{H}$ & July - September & J J 199488 \\
\hline Commelina caroliniana Walter & $\mathrm{H}$ & July - September & J J 198252 \\
\hline Cyanotis axillaris (L.) D. Don ex Sweet & $\mathrm{H}$ & July - September & J J 198237 \\
\hline Cyanotis cristata (L.) D.Don & $\mathrm{H}$ & July - September & 198032 \\
\hline Cyanotis fasciculata (B. Heyne ex Roth) Schult. \& Schult. f. & $\mathrm{H}$ & August - September & J J 198008 \\
\hline \multicolumn{4}{|l|}{ Convolvulaceae } \\
\hline Argyreia cymosa (Roxb.) Sweet & C & November - December & J J 199344 \\
\hline Convolvulus arvensis $\mathrm{L}$. & C & July - August & 199292 \\
\hline
\end{tabular}


Table 1. Continued.

\begin{tabular}{|c|c|c|c|}
\hline Taxa & Habit & Flowers & Field No. \\
\hline Evolvulus alsinoides (L.) L. & $\mathrm{H}$ & July-September & J J 198016 \\
\hline Ipomoea aquatica Forssk. & $\mathrm{H}$ & July - September & 199480 \\
\hline Ipomoea cairica (L.) Sweet & c & July - September & 199365 \\
\hline Ipomoea carnea Jacq. & s & July-October & 198279 \\
\hline Ipomoea eriocarpa R. Br. & C & November - December & 199379 \\
\hline Ipomoea hederifolia L. & c & November - December & 199309 Jנ \\
\hline Ipomoea nil (L.) Roth & C & August - September & 198229 \\
\hline Ipomoea obscura (L.) Ker Gawl & C & September - December & 198235 \\
\hline Ipomoea grandifolia (Dammer) O'Donell & C & September - December & 198168 \\
\hline Ipomoea sindica Stapf & c & September - December & 199466 \\
\hline Ipomoea violacea $\mathrm{L}$. & C & September - December & 199345 \\
\hline Merremia emarginata (Burm.f.) Hallier f. & $\mathrm{H}$ & September - December & 198267 \\
\hline Rivea hypocrateriformis (Desr.) Choisy & C & July-December & 198124 \\
\hline \multicolumn{4}{|l|}{ Cucurbitaceae } \\
\hline Citrullus colocynthis (L.) Schrad. & C & July - October & J J 199306 \\
\hline Citrullus lanatus (Thunb.) Matsum. \& Nakai & C & July - October & 199413 \\
\hline Coccinia grandis (L.) Voigt. & $\mathrm{C}$ & September - December & 198187 \\
\hline Corallocarpus epigaeus (Rottler) Benth. \& Hook. f. & C & September-October & J 198181 \\
\hline Cucumis prophetarum L. & C & September - December & 199299 \\
\hline Cucumis sativus $\mathrm{L}$. & C & September - December & J 198180 \\
\hline Diplocyclos palmatus (L.) C. Jeffrey & C & September - December & J 198118 \\
\hline Momordica charantia L. & C & July - October & J J 199213 \\
\hline Momordica cymbalaria Hook.f. & C & July-August & J J 199261 \\
\hline Momordica dioica Roxb. ex Willd. & C & July-October & J J 199245 \\
\hline Mukia maderaspatana (L.) M. Roem. & C & September - October & J 198024 \\
\hline \multicolumn{4}{|l|}{ Cuscutaceae } \\
\hline Cuscuta reflexa Roxb. & C & December - January & J J 199274 \\
\hline \multicolumn{4}{|l|}{ Cyperaceae } \\
\hline Bulbostylis barbata (Rottb.) C.B. Clarke & $\mathrm{H}$ & September-October & 198042 \\
\hline Cyperus alulatus J. Kern & $\mathrm{H}$ & July - December & 198066 \\
\hline Cyperus compressus L. & $\mathrm{H}$ & July-December & 198283 נJ \\
\hline Cyperus difformis L. & H & July - December & 198206 \\
\hline Cyperus digitatus Roxb. & $\mathrm{H}$ & July - December & 198215 \\
\hline Cyperus distans L. f. & $\mathrm{H}$ & August - October & J 198217 \\
\hline Cyperus exaltatus Retz. & $\mathrm{H}$ & August - December & J J J 199396 \\
\hline Cyperus iria L. & $\mathrm{H}$ & July - December & J J 198036 \\
\hline Cyperus nutans var. eleusinoides (Kunth) R.W. Haines & $\mathrm{H}$ & July - December & 198248 \\
\hline Cyperus pilosus Vahl & $\mathrm{H}$ & July - September & J J 198096 \\
\hline Cyperus rotundus L. & $\mathrm{H}$ & July - December & J 198214 \\
\hline Cyperus tenuispica Steud. & $\mathrm{H}$ & August-October & 198097 \\
\hline Eleocharis atropurpurea (Retz.) J. Presl. \& C. Presl. & $\mathrm{H}$ & November - December & 199319 \\
\hline Eleocharis geniculata (L.) Roem. \& Schult. & $\mathrm{H}$ & August - December & J 198101 \\
\hline Fimbristylis complanata (Retz.) Link & $\mathrm{H}$ & July-October & 198093 \\
\hline Fimbristylis dichotoma (L.) Vahl & $\mathrm{H}$ & July - December & J 198292 \\
\hline Fimbristylis ferruginea (L.) Vahl & $\mathrm{H}$ & August-October & J 198219 \\
\hline Fimbristylis ovata (Burm. f.) J. Kern & $\mathrm{H}$ & August - October & J J 198294 \\
\hline Fimbristylis quinquangularis (Vahl) Kunth & $\mathrm{H}$ & September-October & J J 198089 \\
\hline Fimbristylis tenera Schult. & $\mathrm{H}$ & September-October & J 198041 \\
\hline Fuirena cuspidata (Roth) Kunth & $\mathrm{H}$ & August - October & 198246 \\
\hline Kyllinga brevifolia Rottb. & $\mathrm{H}$ & August - September & 198035 \\
\hline Lipocarpha squarrosa (L.) Goetgheb. & $\mathrm{H}$ & August - September & 198203 \\
\hline Mariscus squarrosus (L.) C.B. Clarke & $\mathrm{H}$ & August - September & 198037 \\
\hline Pycreus flavidus (Retz.) T. Koyama & $\mathrm{H}$ & August - September & 198218 \\
\hline Pycreus polystachyos (Rottb.) P. Beauv. & $\mathrm{H}$ & August - September & 199493 \\
\hline Schoenoplectiella lateriflora (J.F. Gmel) Lye & $\mathrm{H}$ & July - September & J J 199395 \\
\hline Schoenoplectiella roylei (Nees) Lye & $\mathrm{H}$ & September-October & * \\
\hline Schoenoplectiella supina (L.) Lye & $\mathrm{H}$ & July - September & 199397 \\
\hline Scleria parvula Steud. & $\mathrm{H}$ & July - September & J J 198284 \\
\hline \multicolumn{4}{|l|}{ Eriocaulaceae } \\
\hline Eriocaulon quinquangulare L. & $\mathrm{H}$ & August - September & 198202 \\
\hline
\end{tabular}


Table 1. Continued.

\begin{tabular}{|c|c|c|c|}
\hline Taxa & Habit & Flowers & Field No. \\
\hline \multicolumn{4}{|l|}{ Euphorbiaceae } \\
\hline Acalypha indica L. & $\mathrm{H}$ & August - September & 198149 \\
\hline Chrozophora rottleri (Geiseler) A. Juss.ex Spreng. & $\mathrm{H}$ & August - September & 199427 נر \\
\hline Croton bonplandianus Baill. & $\mathrm{H}$ & August-October & 198264 \\
\hline Euphorbia antiquorum L. & s & July - September & * \\
\hline Euphorbia cristata B. Heyne ex Roth & $\mathrm{H}$ & July - September & 198161 \\
\hline Euphorbia heterophylla L. & $\mathrm{H}$ & August - September & 198157 \\
\hline Euphorbia heyneana Spreng. & $\mathrm{H}$ & November - December & 199384 \\
\hline Euphorbia hirta L. & $\mathrm{H}$ & August - September & 198011 \\
\hline Euphorbia hypericifolia L. & $\mathrm{H}$ & July - August & 198010 \\
\hline Euphorbia indica Lam. & $\mathrm{H}$ & August - September & 198274 \\
\hline Euphorbia notoptera Boiss. & $\mathrm{H}$ & August - September & 198071 \\
\hline Euphorbia prostrata Aiton & $\mathrm{H}$ & August - September & 199229 \\
\hline Euphorbia thymifolia L. & $\mathrm{H}$ & August - September & 198275 \\
\hline Euphorbia tirucalli L. & s & December - February & 199414 \\
\hline Flueggea leucopyrus Willd. & $\mathrm{s}$ & July-December & 198067 \\
\hline Jatropha curcas L. & s & December - February & 199249 \\
\hline Jatropha gossypiifolia L. & s & July - October & J J 199207 \\
\hline Phyllanthus amarus Schumach. \& Thonn. & $\mathrm{H}$ & July-October & 198128 \\
\hline Phyllanthus debilis Klein ex Willd., & $\mathrm{H}$ & August - December & J J 199429 \\
\hline Phyllanthus maderaspatensis L. & $\mathrm{H}$ & August - December & J 198005 \\
\hline Phyllanthus reticulatus Poir. & s & July - October & 198200 \\
\hline Phyllanthus simplex Retz. & $\mathrm{H}$ & July-October & J J 199216 \\
\hline Ricinus communis $\mathrm{L}$. & s & October - December & J 194803 \\
\hline Tragia plukenetii Radcl.-Sm. & $\mathrm{H}$ & August-October & J 198154 \\
\hline \multicolumn{4}{|l|}{ Fabaceae } \\
\hline Abrus precatorius $\mathrm{L}$. & c & Almost throughout the year & J J 199390 \\
\hline Aeschynomene indica $\mathrm{L}$. & s & September - October & J J 198231 \\
\hline Alysicarpus glumaceus (Vahl) DC. & $\mathrm{H}$ & September-October & 198173 \\
\hline Alysicarpus heyneanus Wight \& Arn. & $\mathrm{H}$ & July - September & 199322 \\
\hline Alysicarpus monilifer (L.) DC. & $\mathrm{H}$ & July - September & J J 199451 \\
\hline Alysicarpus scariosus (Rottl. ex Spreng.) Graham ex Thwaites & $\mathrm{H}$ & September - October & 199394 \\
\hline Alysicarpus tetragonolobus Edgew. & $\mathrm{H}$ & September - October & 198085 \\
\hline Alysicarpus vaginalis (L.) DC. var. nummularifolius Miq. & $\mathrm{H}$ & September - October & J J 198009 \\
\hline Butea monosperma (Lam.) Taub. & $\mathrm{T}$ & February - March & * \\
\hline Canavalia africana Dunn & C & July - December & J J 199343 \\
\hline Canavalia ensiformis (L.) DC. & c & July-December & 199457 \\
\hline Crotalaria calycina Schrank & $\mathrm{H}$ & September - October & 198153 \\
\hline Crotalaria filipes Benth & $\mathrm{H}$ & September - October & 198256 \\
\hline Crotalaria hebecarpa (DC.) Rudd & $\mathrm{H}$ & September - December & 198086 \\
\hline Crotalaria juncea L. & $\mathrm{H}$ & November - December & 199399 \\
\hline Crotalaria orixensis Willd. & $\mathrm{H}$ & September-October & 198162 \\
\hline Crotalaria pallida Aiton. & $\mathrm{H}$ & July - October & 199473 \\
\hline Crotalaria vestita Baker & $\mathrm{H}$ & September-October & J 198122 \\
\hline Desmodium dichotomum (Willd.) DC. & $\mathrm{H}$ & September-October & 198174 \\
\hline Desmodium gangeticum (L.) DC. & s & September-October & J J 199447 \\
\hline Desmodium heterocarpon (L.) DC. & s & September-October & 198129 \\
\hline Desmodium triflorum (L.) DC. & $\mathrm{H}$ & July - September & 199497 \\
\hline Erythrina variegata $\mathrm{L}$. & $\mathrm{T}$ & March - April & 194804 \\
\hline Indigofera coerulea Roxb. & s & July - August & 199296 \\
\hline Indigofera cordifolia B. Heyne ex Roth & $\mathrm{H}$ & September - December & 198018 \\
\hline Indigofera linifolia (L.f.) Retz. & $\mathrm{H}$ & September - December & 198025 \\
\hline Indigofera prostrata Willd. & $\mathrm{H}$ & August-October & * \\
\hline Indigofera tinctoria $\mathrm{L}$. & $\mathrm{s}$ & July - October & J J 199291 \\
\hline Indigofera trifoliata L. & $\mathrm{H}$ & September - December & 198190 \\
\hline Lablab purpureus (L.) Sweet & c & July - September & 199356 \\
\hline Rhynchosia capitata (B. Heyne ex Roth) DC. & c & September - December & 198198 \\
\hline Rhynchosia minima (L.) DC. & c & September - December & 198120 \\
\hline Stylosanthes fruticosa (Retz.) Alston & S & September - October & J J 198092 \\
\hline Stylosanthes viscosa (L.) Sw. & $\mathrm{s}$ & September-October & 198258 \\
\hline
\end{tabular}


Table 1. Continued.

\begin{tabular}{|c|c|c|c|}
\hline Taxa & Habit & Flowers & Field No. \\
\hline Tephrosia purpurea (L.) Pers. & $\mathrm{s}$ & September - October & 198022 \\
\hline Tephrosia senticosa (L.) Pers. & $\mathrm{s}$ & September - December & 198192 \\
\hline Tephrosia villosa (L.) Pers. & $\mathrm{s}$ & July - October & 198226 \\
\hline Vigna aconitifolia (Jacq.) Morechal & C & August - September & J J 198167 \\
\hline Vigna indica T.M. Dixit, K.V. Bhat \& S.R. Yadav & C & August - September & 198019 Jנ \\
\hline Vigna trilobata (L.) Verdc. & C & August - September & 198164 \\
\hline Zornia gibbosa Span. & $\mathrm{H}$ & August - September & 198001 \\
\hline \multicolumn{4}{|l|}{ Gentianaceae } \\
\hline Canscora diffusa (Vahl) R. Br. & $\mathrm{H}$ & August - September & 198220 \\
\hline Enicostema axillare (Lam.) Raynal & $\mathrm{H}$ & August - September & 198073 נJ \\
\hline Exacum pumilum Griseb. & $\mathrm{H}$ & August - September & 198106 \\
\hline \multicolumn{4}{|l|}{ Geraniaceae } \\
\hline Monsonia senegalensis Guill. \& Perr. & $\mathrm{H}$ & July - August & 199293 \\
\hline \multicolumn{4}{|l|}{ Hydrocharitaceae } \\
\hline Ottelia alismoides (L.) Pers. & $\mathrm{H}$ & October - December & 199354 \\
\hline \multicolumn{4}{|l|}{ Lamiaceae } \\
\hline Hyptis suaveolens (L.) Poit. & $\mathrm{H}$ & July - September & J J 198210 \\
\hline Lavandula bipinnata (Roth) Kuntze & $\mathrm{H}$ & August - September & 198105 \\
\hline Leonotis nepetifolia (L.) R. Br. & s & November - December & 199358 \\
\hline Leucas aspera (Willd.) Link & $\mathrm{H}$ & July - October & J J 198244 \\
\hline Leucas lavandulifolia Sm. & $\mathrm{H}$ & July - October & J J 198175 \\
\hline Leucas longifolia Benth. & $\mathrm{H}$ & July - October & J J J 198057 \\
\hline Ocimum tenuiflorum L. & $\mathrm{H}$ & July - October & J J 198100 \\
\hline Orthosiphon pallidus Royle ex Benth. & $\mathrm{H}$ & July - September & J J 198151 \\
\hline \multicolumn{4}{|l|}{ Lentibulariaceae } \\
\hline Utricularia bifida L. & $\mathrm{H}$ & November - December & 199375 \\
\hline \multicolumn{4}{|l|}{ Liliaceae } \\
\hline Aloe vera (L.) Burm.f. & $\mathrm{H}$ & December - March & J J 199492 \\
\hline Asparagus racemosus Willd. & C & October - February & 199286 \\
\hline Chlorophytum laxum R. Br. & $\mathrm{H}$ & July - September & J J 199285 \\
\hline Chlorophytum tuberosum (Roxb.) Baker & $\mathrm{H}$ & July - September & 199252 \\
\hline Ledebouria hyacinthina (L.f.) Jessop & $\mathrm{H}$ & July - September & 199217 \\
\hline \multicolumn{4}{|l|}{ Lobeliaceae } \\
\hline Lobelia alsinoides Lam. & $\mathrm{H}$ & August - October & 199376 \\
\hline \multicolumn{4}{|l|}{ Lythraceae } \\
\hline Ammannia baccifera $\mathrm{L}$. & $\mathrm{H}$ & November - December & J J 198241 \\
\hline Ammannia multiflora Roxb. & $\mathrm{H}$ & September - October & 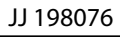 \\
\hline \multicolumn{4}{|l|}{ Malvaceae } \\
\hline Abutilon hirtum (Lam.) Sweet & s & July - October & 199270 \\
\hline Abutilon indicum (L.) Sweet & $\mathrm{s}$ & August - December & 198230 \\
\hline Abutilon pannosum (G. Forst.) Schltdl. & S & September - December & J J 198183 \\
\hline Hibiscus caesius Garcke & $\mathrm{H}$ & October - December & J J J 199359 \\
\hline Hibiscus lobatus (Murray) Kuntze. & $\mathrm{H}$ & December - January & 199280 \\
\hline Malachra capitata (L.) L. & $\mathrm{s}$ & December - January & 199462 \\
\hline Malvastrum coromandelianum (L.) Garcke & s & July - September & J J 198160 \\
\hline Sida acuta Burm. f. & s & September - December & J J 198261 \\
\hline Sida rhombifolia L. & S & October - December & J J J 194805 \\
\hline Sida spinosa L. & s & September - December & J J 198289 \\
\hline Thespesia populnea (L.) Sol. ex Correa & $\mathrm{T}$ & August - December & J J 194806 \\
\hline \multicolumn{4}{|l|}{ Martyniaceae } \\
\hline Martynia annua L. & $\mathrm{H}$ & August - September & J J 198115 \\
\hline \multicolumn{4}{|l|}{ Menispermaceae } \\
\hline Cocculus hirsutus (L.) W. Theob. & c & Frequently throughout the year & J J 198199 \\
\hline Tinospora cordifolia (Willd.) Miers ex Hook. f. \& Thoms & C & July - October & 198201 \\
\hline \multicolumn{4}{|l|}{ Mimosaceae } \\
\hline Acacia campbelii Arn. & $\mathrm{T}$ & August - December & J J 199329 \\
\hline Acacia catechu (L.f.) Willd. & T & August - October & 198194 \\
\hline Acacia eburnea (L.f.) Willd. & $\mathrm{T}$ & September - December & 194807 \\
\hline Acacia leucophloea (Roxb.) Willd. & $\mathrm{T}$ & September - December & J J 198058 \\
\hline Acacia nilotica subsp. cupressiformis (J.L. Stewart) Ali \& Faruqui & $\mathrm{T}$ & August - December & 194808 \\
\hline
\end{tabular}


Table 1. Continued.

\begin{tabular}{|c|c|c|c|}
\hline Taxa & Habit & Flowers & Field No. \\
\hline Acacia nilotica subsp. indica (Benth.) Brenan & $T$ & September-October & J J 198140 \\
\hline Acacia tomentosa Willd. & $\mathrm{T}$ & September - December & 199273 \\
\hline Dichrostachys cinerea var. indica Brenan \& Brummit & $S$ & August - December & 199237 \\
\hline Leucaena leucocephala (Lam.) de Wit & $\mathrm{T}$ & August - December & 194809 \\
\hline Mimosa hamata Willd. & $S$ & August - December & 198099 \\
\hline Mimosa pudica L. & $\mathrm{H}$ & August - December & 194810 \\
\hline Pithecellobium dulce (Roxb.) Benth. & $\mathrm{T}$ & February - June & J J 199455 \\
\hline Prosopis juliflora (Sw.) DC. & $\mathrm{T}$ & February - June & J 194811 \\
\hline \multicolumn{4}{|l|}{ Molluginaceae } \\
\hline Glinus lotoides L. & $\mathrm{H}$ & August - September & 199240 \\
\hline Mollugo nudicaulis Lam. & $\mathrm{H}$ & September - October & 198028 \\
\hline Mollugo stricta $\mathrm{L}$. & $\mathrm{H}$ & September - December & 199393 \\
\hline \multicolumn{4}{|l|}{ Moraceae } \\
\hline Ficus benghalensis $\mathrm{L}$. & $\mathrm{T}$ & February - June & 194812 \\
\hline Ficus hispida L. f. & $\mathrm{T}$ & July - August & 194813 \\
\hline Ficus microcarpa L. f. & $\mathrm{T}$ & July-August & 199272 \\
\hline Ficus racemosa $\mathrm{L}$. & $\mathrm{T}$ & July - August & 199250 \\
\hline Ficus religiosa $\mathrm{L}$. & $\mathrm{T}$ & July - August & J 194814 \\
\hline \multicolumn{4}{|l|}{ Nyctaginaceae } \\
\hline Boerhavia erecta L. & $\mathrm{H}$ & September - October & 199421 \\
\hline Boerhavia diffusa $\mathrm{L}$. & $\mathrm{H}$ & September-October & 198077 JנJ \\
\hline Mirabilis jalapa L. & $\mathrm{H}$ & Frequently throughout the year & 199486 \\
\hline \multicolumn{4}{|l|}{ Onagraceae } \\
\hline Ludwigia hyssopifolia (G.Don) Exell & $\mathrm{H}$ & September-October & 198211 \\
\hline \multicolumn{4}{|l|}{ Oxalidaceae } \\
\hline Biophytum sensitivum (L.) DC. & $\mathrm{H}$ & September-October & 198033 \\
\hline Oxalis corniculata L. & $\mathrm{H}$ & Almost throughout the year & 199366 \\
\hline \multicolumn{4}{|l|}{ Papaveraceae } \\
\hline Argemone mexicana L. & $\mathrm{H}$ & Almost throughout the year & 198300 \\
\hline \multicolumn{4}{|l|}{ Passifloraceae } \\
\hline Passiflora foetida L. & C & August - December & 194815 \\
\hline \multicolumn{4}{|l|}{ Pedaliaceae } \\
\hline Sesamum laciniatum Klein. ex Willd. & $\mathrm{H}$ & August - September & 198023 \\
\hline Sesamum indicum $\mathrm{L}$. & $\mathrm{H}$ & August - September & 198146 \\
\hline \multicolumn{4}{|l|}{ Periplocaceae } \\
\hline Cryptolepis buchananii Roem. \& Schult. & $\mathrm{s}$ & July - September & 198233 נJ \\
\hline Cryptostegia grandiflora R. Br. & s & July-September & 198056 \\
\hline Hemidesmus indicus (L.) Schult. & C & July - September & 199479 \\
\hline \multicolumn{4}{|l|}{ Plumbaginaceae } \\
\hline Plumbago zeylanica L. & $\mathrm{S}$ & September-October & 198232 \\
\hline \multicolumn{4}{|l|}{ Poaceae } \\
\hline Alloteropsis cimicina (L.) Stapf. & $\mathrm{H}$ & July - September & 198127 \\
\hline Andropogon pumilus Roxb. & $\mathrm{H}$ & July - December & 198133 \\
\hline Apluda mutica $\mathrm{L}$. & $\mathrm{H}$ & September - December & 198170 \\
\hline Aristida adscensionis $\mathrm{L}$. & $\mathrm{H}$ & September - December & 199348 \\
\hline Aristida funiculata Trin. \& Rupr. & $\mathrm{H}$ & September - December & 199494 \\
\hline Aristida hystrix L. f. & $\mathrm{H}$ & September - December & 198139 \\
\hline Aristida redacta Stapf. & $\mathrm{H}$ & September - December & 198108 \\
\hline Aristida setacea Retz. & $\mathrm{H}$ & September - December & 199495 \\
\hline Aristida stocksii (Hook. f.) Domin & $\mathrm{H}$ & July - December & 198040 \\
\hline Arundinella tuberculata Munro ex Lisboa & $\mathrm{H}$ & September - November & * \\
\hline Arundo donax L. & $\mathrm{H}$ & September - November & 199334 נر \\
\hline Bothriochloa pertusa (L.) A. Camus & $\mathrm{H}$ & September - December & 198029 נر \\
\hline Brachiaria distachya (L.) Stapf. & $\mathrm{H}$ & September - November & 198117 \\
\hline Brachiaria eruciformis (Sm.) Griseb. & $\mathrm{H}$ & July - September & 198165 \\
\hline Brachiaria ramosa (L.) Stapf. & $\mathrm{H}$ & July - September & (נر 198176 \\
\hline Cenchrus setigerus Vahl & $\mathrm{H}$ & July - September & 198184 \\
\hline Chionachne gigantea (J. Koenig) Veldkamp & $\mathrm{H}$ & July - September & 198259 \\
\hline Chloris gayana Kunth ex Stapf & $\mathrm{H}$ & July - September & 198135 \\
\hline Chrysopogon fulvus (Spreng.) Chiov. & $\mathrm{H}$ & August - September & 198143 נر \\
\hline
\end{tabular}


Table 1. Continued.

\begin{tabular}{|c|c|c|c|}
\hline Taxa & Habit & Flowers & Field No. \\
\hline Coix lacryma-jobi L. & $\mathrm{H}$ & July - September & J J 199368 \\
\hline Cymbopogon martini (Roxb.) S. Watson & $\mathrm{H}$ & July - October & 198265 \\
\hline Cynodon dactylon (L.) Pers. & $\mathrm{H}$ & August - October & J J 199224 \\
\hline Dactyloctenium aegyptiacum (L.) Willd. & $\mathrm{H}$ & August - October & J J 198260 \\
\hline Dactyloctenium aristatum Link & $\mathrm{H}$ & August - October & J J 198048 \\
\hline Dichanthium foveolatum (Delile) Roberty & $\mathrm{H}$ & August - October & J J 198185 \\
\hline Digitaria ciliaris (Retz.) Koeler & $\mathrm{H}$ & August - October & J J 198044 \\
\hline Digitaria stricta Roth ex Roem. \& Schult. & $\mathrm{H}$ & August - October & 198081 \\
\hline Digitaria ternata (A. Rich.) Stapf. & $\mathrm{H}$ & September - December & J J 199496 \\
\hline Dinebra retroflexa (Vahl) Panz. & $\mathrm{H}$ & August - October & J J 198269 \\
\hline Echinochloa colonum (L.) Link & $\mathrm{H}$ & August - September & J J 198046 \\
\hline Eleusine indica (L.) Gaertn. & $\mathrm{H}$ & July - September & J J 198227 \\
\hline Eragrostiella bifaria (Vahl) & $\mathrm{H}$ & July - September & J 198111 \\
\hline Eragrostiella brachyphylla (Stapf) Bor & $\mathrm{H}$ & July - September & 198043 \\
\hline Eragrostis gangetica (Roxb.) Steud & $\mathrm{H}$ & September - October & J J 198112 \\
\hline Eragrostis pilosa (L.) P. Beauv. & $\mathrm{H}$ & September - October & J J 198045 \\
\hline Eragrostis tenella (L.) P. Beauv. ex Roem. \& Schult. & $\mathrm{H}$ & August - October & J J 198268 \\
\hline Eragrostis unioloides (Retz.) Nees ex Steud. & $\mathrm{H}$ & August - October & J J 199382 \\
\hline Eragrostis viscosa (Retz.) Trin. & $\mathrm{H}$ & September - October & J J 199336 \\
\hline Heteropogon contortus (L.) P. Beauv. ex Roem. \& Schult. & $\mathrm{H}$ & September - October & J J 198079 \\
\hline Heteropogon triticeus (R. Br.) Stapf ex Craib & $\mathrm{H}$ & September - October & J J 199370 \\
\hline Isachne borii Hemadri & $\mathrm{H}$ & September - October & * \\
\hline Ischaemum afrum (J.F. Gmel.) Dandy & $\mathrm{H}$ & July - September & J J 198186 \\
\hline Iseilema anthephoroides Hack. & $\mathrm{H}$ & September - October & 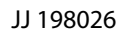 \\
\hline Hackelochloa granularis (L.) Kuntze & $\mathrm{H}$ & September - October & J J 198014 \\
\hline Lophopogon tridentatus (Roxb.) Hack. & $\mathrm{H}$ & August - October & J J 198038 \\
\hline Melanocenchris jacquemontii Jaub. \& Spach & $\mathrm{H}$ & August - October & J J 198039 \\
\hline Oropetium roxburghianum (Steud.) S.M. Phillips & $\mathrm{H}$ & August - September & J J 199226 \\
\hline Oropetium thomaeum (L. f.) Trin. & $\mathrm{H}$ & August - September & J J 198126 \\
\hline Oropetium villosulum Stapf ex Bor & $\mathrm{H}$ & August - September & J J 199419 \\
\hline Oryza sativa $\mathrm{L}$. & $\mathrm{H}$ & July-September & 198285 \\
\hline Panicum hippothrix K.Schum. ex Engl. & $\mathrm{H}$ & July - October & J J 199498 \\
\hline Panicum curviflorum Hornem & $\mathrm{H}$ & September - October & J J 198087 \\
\hline Paspalidium flavidum (Retz.) A. Camus & $\mathrm{H}$ & August - October & J J 198027 \\
\hline Pennisetum pedicellatum Trin. & $\mathrm{H}$ & July - September & J J 199499 \\
\hline Pennisetum purpureum Schumach. & $\mathrm{H}$ & July - September & J J 199271 \\
\hline Sehima ischaemoides Forssk. & $\mathrm{H}$ & July - October & J J 198137 \\
\hline Sehima sulcatum (Hack.) A. Camus & $\mathrm{H}$ & July - October & J J 199392 \\
\hline Setaria intermedia Roem. \& Schult. & $\mathrm{H}$ & July - September & J J 198136 \\
\hline Setaria pumila (Poir.) Roem. \& Schult. & $\mathrm{H}$ & July - October & J J 198047 \\
\hline Spodiopogon rhizophorus (Steud.) Pilg. & $\mathrm{H}$ & July - October & * \\
\hline Sporobolus coromandelianus (Retz.) Kunth & $\mathrm{H}$ & July - October & * \\
\hline Sporobolus indicus (L.) R. Br. & $\mathrm{H}$ & July - October & J J 199452 \\
\hline Tetrapogon tenellus (J. Koenig ex Roxb.) Chiov. & $\mathrm{H}$ & July - October & J J 198225 \\
\hline Themeda laxa (Andersson) A. Camus & $\mathrm{H}$ & July - December & J J 199369 \\
\hline Tragus mongolorum Ohwi & $\mathrm{H}$ & July - October & J J 198074 \\
\hline Tripogon bromoides Roth & $\mathrm{H}$ & July - October & J J 199437 \\
\hline Tripogon jacquemontii Stapf & $\mathrm{H}$ & July - October & J J 198249 \\
\hline \multicolumn{4}{|l|}{ Polygalaceae } \\
\hline Polygala arvensis Willd. & $\mathrm{H}$ & July - September & J J 198034 \\
\hline Polygala erioptera DC. Prodr. & $\mathrm{H}$ & August - September & J 198084 \\
\hline \multicolumn{4}{|l|}{ Polygonaceae } \\
\hline Antigonon leptopus Hook. \& Arn. & $\mathrm{C}$ & Almost throughout the year & J J 199487 \\
\hline Persicaria glabra (Willd.) M. Gomez & $\mathrm{H}$ & August - September & J J 199453 \\
\hline Polygonum plebeium R. Br. & $\mathrm{H}$ & July - September & J J 199227 \\
\hline \multicolumn{4}{|l|}{ Pontederiaceae } \\
\hline Eichhornia crassipes (Mart.) Solms & $\mathrm{H}$ & Almost throughout the year & * \\
\hline \multicolumn{4}{|l|}{ Portulacaceae } \\
\hline Portulaca oleracea L. & $\mathrm{H}$ & July - September & J J 198017 \\
\hline Portulaca tuberosa Roxb. & $\mathrm{H}$ & July - September & J J 198204 \\
\hline Portulaca quadrifida L. & $\mathrm{H}$ & August - September & J J 199430 \\
\hline
\end{tabular}


Table 1. Continued.

\begin{tabular}{|c|c|c|c|}
\hline Taxa & Habit & Flowers & Field No. \\
\hline \multicolumn{4}{|l|}{ Rhamnaceae } \\
\hline Ziziphus mauritiana Lam. & $\mathrm{S}$ & September - December & J J 198068 \\
\hline Ziziphus xylopyrus (Retz.) Willd. & $\mathrm{S}$ & August - December & J J 199386 \\
\hline \multicolumn{4}{|l|}{ Rubiaceae } \\
\hline Benkara malabarica (Lam.) Tirveng. & $\mathrm{S}$ & June - October & 198223 \\
\hline Dentella repens (L.) J.R. Forst. \& G. Forst. & $\mathrm{H}$ & July - August & J 199225 \\
\hline Kohautia aspera (B. Heyne ex Roth) Bremek. & $\mathrm{H}$ & August - November & * \\
\hline Morinda pubescens J.E. Sm. & $\mathrm{T}$ & Almost throughour the year & J J 198055 \\
\hline Neanotis montholonii (Hook. f.) W.H. Lewis & $\mathrm{H}$ & September - October & J J 198159 \\
\hline Oldenlandia corymbosa $\mathrm{L}$. & $\mathrm{H}$ & September - October & 198240 \\
\hline Oldenlandia diffusa (Willd.) Roxb. & $\mathrm{H}$ & September - October & J J 199468 \\
\hline Oldenlandia umbellata $\mathrm{L}$. & $\mathrm{H}$ & August - December & * \\
\hline Spermacoce articularis L.f. & $\mathrm{H}$ & September - October & J J 198064 \\
\hline Spermacoce pusilla Wall. & $\mathrm{H}$ & September - October & J J 198003 \\
\hline \multicolumn{4}{|l|}{ Santalaceae } \\
\hline Santalum album L. & $\mathrm{T}$ & August - October & JJ 198114 \\
\hline \multicolumn{4}{|l|}{ Sapindaceae } \\
\hline Cardiospermum halicacabum L. & $\mathrm{C}$ & Almost throughout the year & J J 198130 \\
\hline \multicolumn{4}{|l|}{ Scrophulariaceae } \\
\hline Bacopa monnieri (L.) Wettst. & $\mathrm{H}$ & July - August & 199290 \\
\hline Limnophila heterophylla (Roxb.) Benth. & $\mathrm{H}$ & November - December & J J 199374 \\
\hline Lindernia antipoda (L.) Alston & $\mathrm{H}$ & November - December & J J 199483 \\
\hline Lindernia ciliata (Colsm.) Pennell & $\mathrm{H}$ & November - December & J J 199378 \\
\hline Lindernia crustacea (L.) F. Muell. & $\mathrm{H}$ & November - December & J J 199484 \\
\hline Sopubia delphinifolia D. Don & $\mathrm{H}$ & August - September & J 198061 \\
\hline Stemodia viscosa Roxb. & $\mathrm{H}$ & November - December & J J 199373 \\
\hline Striga asiatica (L.) Kuntze & $\mathrm{H}$ & August - September & J J 198006 \\
\hline Striga densiflora (Benth.) Benth & $\mathrm{H}$ & August - September & J J 198004 \\
\hline Striga gesnerioides (Willd.) Vatke & $\mathrm{H}$ & August - September & 198069 \\
\hline Verbascum chinense (L.) Santapau & $\mathrm{H}$ & July - August & J 199230 \\
\hline \multicolumn{4}{|l|}{ Solanaceae } \\
\hline Datura inoxia Mill. & $\mathrm{H}$ & September - December & J 198278 \\
\hline Datura metel L. & $\mathrm{H}$ & September - December & J J 199481 \\
\hline Datura ferox L. & $\mathrm{H}$ & August - September & J J 199409 \\
\hline Physalis angulata $\mathrm{L}$. & $\mathrm{H}$ & July - August & J 198182 \\
\hline Solanum erianthum D. Don & $\mathrm{S}$ & July - August & 199247 Jנ \\
\hline Solanum incanum $\mathrm{L}$. & $\mathrm{S}$ & July - September & J J 199211 \\
\hline Solanum americanum Mill. & $\mathrm{S}$ & July - October & J J 199482 \\
\hline Solanum virginianum $\mathrm{L}$. & $\mathrm{S}$ & July - October & 199214 \\
\hline \multicolumn{4}{|l|}{ Tiliaceae } \\
\hline Corchorus fascicularis Lam. & $\mathrm{H}$ & September - December & J J 198065 \\
\hline Corchorus olitorius L. & $\mathrm{H}$ & September - December & J J 198121 \\
\hline Corchorus trilocularis L. & $\mathrm{H}$ & September - December & 198243 \\
\hline Grewia tenax (Forssk.) Fiori & $\mathrm{S}$ & April - September & * \\
\hline Grewia villosa Willd. & $\mathrm{S}$ & August - December & JJ 199326 \\
\hline Triumfetta malabarica Koen. ex Rottb. & $\mathrm{S}$ & September - October & JJ 198155 \\
\hline \multicolumn{4}{|l|}{ Typhaceae } \\
\hline Typha angustifolia L. & $\mathrm{H}$ & July - September & 199367 \\
\hline \multicolumn{4}{|l|}{ Verbenaceae } \\
\hline Clerodendrum phlomidis L. f. & $\mathrm{S}$ & July - December & J J 199253 \\
\hline Lantana camara L. & $\mathrm{S}$ & Frequently throughout the year & J J 199485 \\
\hline Phyla nodiflora (L.) Greene & $\mathrm{H}$ & July - August & 199219 \\
\hline Vitex negundo L. & $\mathrm{S}$ & July - September & J J 199206 \\
\hline \multicolumn{4}{|l|}{ Vitaceae } \\
\hline Cissus arnottiana Shetty \& P. Singh & $\mathrm{S}$ & Irregularly throughout the year & J 198224 \\
\hline Cissus quadrangularis $\mathrm{L}$. & $\mathrm{C}$ & Almost throughout the year & 194816 \\
\hline \multicolumn{4}{|l|}{ Zygophyllaceae } \\
\hline Balanites aegyptiaca (L.) Delile & $\mathrm{S}$ & July - December & J J 198266 \\
\hline Tribulus terrestris $\mathrm{L}$. & $\mathrm{H}$ & July - December & J 198007 \\
\hline
\end{tabular}


coupled with high probability of extinction of bustards in the near future, the Ministry of Environment and Forests (MoEF), Government of India, has included the Great Indian Bustard under the Species recovery component of the Centrally Sponsored Scheme - Integrated Development of Wildlife Habitats (CSS-IDWH). In this programme, a task force is constituted to prepare an action plan to conserve bustards and their habitats. Hence, this documentation study is important in providing the floristic diversity of the bustard habitat, to continue habitat specific conservation measures.

\section{ACKNOWLEDGEMENTS}

The authors are thankful to Dr. Paramjit Singh, Director, Botanical Survey of India for encouragement and for providing all the facilities and Dr. D. K. Singh, Additional Director for constant support. Sincere thanks to Shri. M. K. Rao, Chief Conservator of Forests, Pune, forest department officials and staff for granting permission and necessary help during the field tours. Our sincere thanks to Mrs. Anne Dayanandan, for critically reviewing the manuscript for language correction and to Dr. P. Dayanandan (Professor, retired), Madras Christian College for providing valuable suggestions. Thanks are due to anonymous reviewers for their valuable comments.

\section{LITERATURE CITED}

Allaby, M. 2006. Biomes of the earth: grasslands. New York: Chelsea House Publishers. 257 pp.

Bhagat, R., V.B. Shimpale, G.G. Potdar and R.B. Deshmukh. 2008. Flora of Baramati. Baramati: Rani B. Bhagat, A/P-Hol (8-Phata). $450 \mathrm{pp}$.

BHL. [2013]. Biodiversity Heritage Library. Accessed at http://www. biodiversitylibrary.org, 25 May 2013.

BirdLife International. 2011. Threatened birds of Asia. The BirdLife International Red Data Book. Cambridge, UK. 3038 pp.

Brummitt, R.K. and C.E. Powell. 2004. Authors of plant names. Kew: Royal Botanic Gardens. 740 pp.

Champion, H.G. and S.K. Seth. 1968. A revised survey of the forest types of India. New Delhi: Manager of Publications. 404 pp.

Cooke, T. 1901. The flora of the Presidency of Bombay. Vol. 1. London: Tailor and Francis. 645 pp.

Cooke, T. 1908. The flora of the Presidency of Bombay. Vol. 2. London: Tailor and Francis. 1083 pp.

Dabadghao, P.M. and K.A. Shankaranarayan. 1973. The grass cover of India. New Delhi: Indian Council of Agricultural Research. 713 pp.

Das Das, S.K. and N.P. Singh. 2006. Additions to the flora of Ahmednagar district (Maharashtra). Journal of Economic and Taxonomic Botany 30(3): 595-602.

Das Das, S.K. and N.P. Singh. 2012. Floristic diversity and conservation of Ujani Wetlands (Maharashtra). Dehra Dun: Bishen Singh Mahendra Pal Singh. 302 pp.

Das Das, S.K. 2012. A contribution to the flora of Solapur District Maharashtra, India. Nelumbo 54: 157-162.

Dinakaran, J., N. Mehta and N.S.R. Krisnayya. 2011. Soil organic carbon dynamics in two functional types of ground cover (grasses and herbaceous) in the tropics. Current Science 101(6): 776-783.

Dutta, S., A.R. Rahmani and Y.V. Jhala. 2011. Running out of time? The Great Indian Bustard Ardeotis nigriceps-status, viability, and conservation strategies. European Journal of Wildlife
Research 57: 615-625. doi: 10.1007/s10344-010-0472-Z

Gaikwad, S.P., R.D. Gore and K.U. Garad. 2012. Additions to the flora of Marathwada region of Maharashtra, India. Journal of Threatened Taxa 4(4): 2515-2523. http://threatenedtaxa. org/viewPDFFile.asp? fname $=0283526$ iv122515-2523. pdf\&typ $=$ jnl\&Imonth $=4$ \&Iyear $=2012$

GBIF. 2013. Global Biodiversity Information Facility. Accessed at http://www.gbif.org, 20 July 2013.

Government of India. 2006. Report of the Task Force on Grasslands and Deserts. New Delhi: Planning Commission. 32 pp.

IUCN. 2014. World Commission on Protected Areas - Temperate Grasslands Specialist Group. International Union for the Conservation of Nature. Accessed at http://www.iucn. org/about/work/programmes/gpap_home/gpap_ biodiversity/gpap_wcpabiodiv/gpap_grasslands, 30 October 2014.

Karthikeyan, S., M. Sanjappa and S. Moorthy. 2009. Flowering plants of India:. dicotyledons. Volume I. Kolkata: Botanical Survey of India. $365 \mathrm{pp}$.

WCSP. 2013. World checklist of selected plant families. Royal Botanic Gardens, Kew. 2013. Accessed at http://apps.kew.org/wcsp/, 18 May 2013.

Lejune, K.D. and T.R. Seastedt. 2001. Centaurea species: the forb that won the west. Conservation Biology 15(6): 1568-1574. doi: 10.1046/j.1523-1739.2001.00242.x

Mooney, H.A. and R.J. Hobbs. 200o. Invasive species in a changing world. Washington, D.C.: Island Press. 457 pp.

Munjpara, S.B., C.N. Pandey and B. Jethva. 2012. Habitat use by the Great Indian Bustard Ardeotis nigriceps (Gruiformes: Otididae) in breeding and non-breeding seasons in Kachchh, Gujarat, India. Journal of Threatened Taxa 5(2): 3654-366o. doi: 10.11609/ JoTT.02757.3654-60

Naik, V.N. 1979. The flora of Osmanabad. Aurangabad: Venus Publishers. 466 pp.

Nancy, D., K. Kowalkowski and C. Throp (eds.). 2013. Grasslandshabitat survival. London: Capstone Global Library Ltd. 32 pp.

Pratibha, P. 2005. National parks and sanctuaries in Maharashtra. Volume I. Mumbai: Bombay Natural History Society. 212 pp.

Pratibha, P. 2005. National parks and sanctuaries in Maharashtra. Volume 2. Mumbai: Bombay Natural History Society. 531 pp.

Pradhan, S.G. and N.P. Singh. 1999. Flora of Ahmednagar District (Maharashtra). Dehra Dun: Bishen Singh Mahendra Pal Singh. $339 \mathrm{pp}$.

Rodgers, W.A. and V.B. Sawarkar. 1988. Vegetation management in wildlife protected areas in India. Aspects of Applied Biology 16: 407-422.

Rahmani, A.R. 1992. Threatened fauna of Indian Grasslands; pp. 143-151, in: K.P. Singh and J.S. Singh (eds.). Tropical ecosystems: ecology and management. New Delhi: Wiley Eastern Ltd.

Rahmani, A. R. 2006. Need to start Project Bustards. Mumbai: Bombay Natural History. 20 pp.

Schlesinger, W.H. 1997. Biogeochemistry: an analysis of global change. San Diego: Academic Press. 588 pp.

Sharma, B.D., S. Karthikeyan and N.P. Singh (eds.). 1996. Flora of Maharashtra state. Monocotyledones. Calcutta: Botanical Survey of India. $794 \mathrm{pp}$.

Shantz, H.L. 1954. The place of grasslands in the Earth's cover of vegetation. Ecology 35(2): 143-145. doi: 10.2307/1931110

Singh, N.P. and S. Karthikeyan (eds.). 200o. Flora of Maharashtra state-Dicotyledones. Volume I. Kolkata: Botanical Survey of India. 898 pp.

Singh, N.P., P. Lakshminarasimhan, S. Karthikeyan and P.V. Prasanna. 2001. Flora of Maharashtra state-Dicotyledones. Volume II. Kolkata: Botanical Survey of India. 1080 pp.

Suryavanshi, R. and M. Bachulkar. 2011. Arboreal flora of Solapur 
Corporation. Journal of Botanical Research 2(1): 8-16.

IPNI. 2013. The international plant names index. Accessed at http:// www.ipni.org/, 20 June 2013.

The Plant List. 2010. Version 1. Accessed at http://www. theplantlist.org/, 18 May 2013.

Tropicos. 2013. Tropicos. Missouri Botanic Garden. Accessed at http://www.tropicos.org/, 25 June 2013.

UNDP. 2012. Fast facts on India's biodiversity, part 2-ecosystems and habitats. United Nations Development Programme. Accessed at http://www.in.undp.org/, 6 January 2015.

Wagner, F.H. 1989. Grazers, past and present; pp. 151-162, in: L.F. Huenneke and H. Mooney (eds.). grassland structure and function: California annual grassland. Dordrecht, The Netherlands: Kluwer Academic Publishers.

Whyte, R.O. 1957. The grassland and fodder resources of India. Indian Council of Agricultural Research Sciences Monograph 22: 1-437.

Authors' contribution statement: JJ collected the data and JJ and JSJ wrote the text and made analysis.

Received: December 2013

Accepted: January 2014

Editorial responsibility: Paul A. Egan
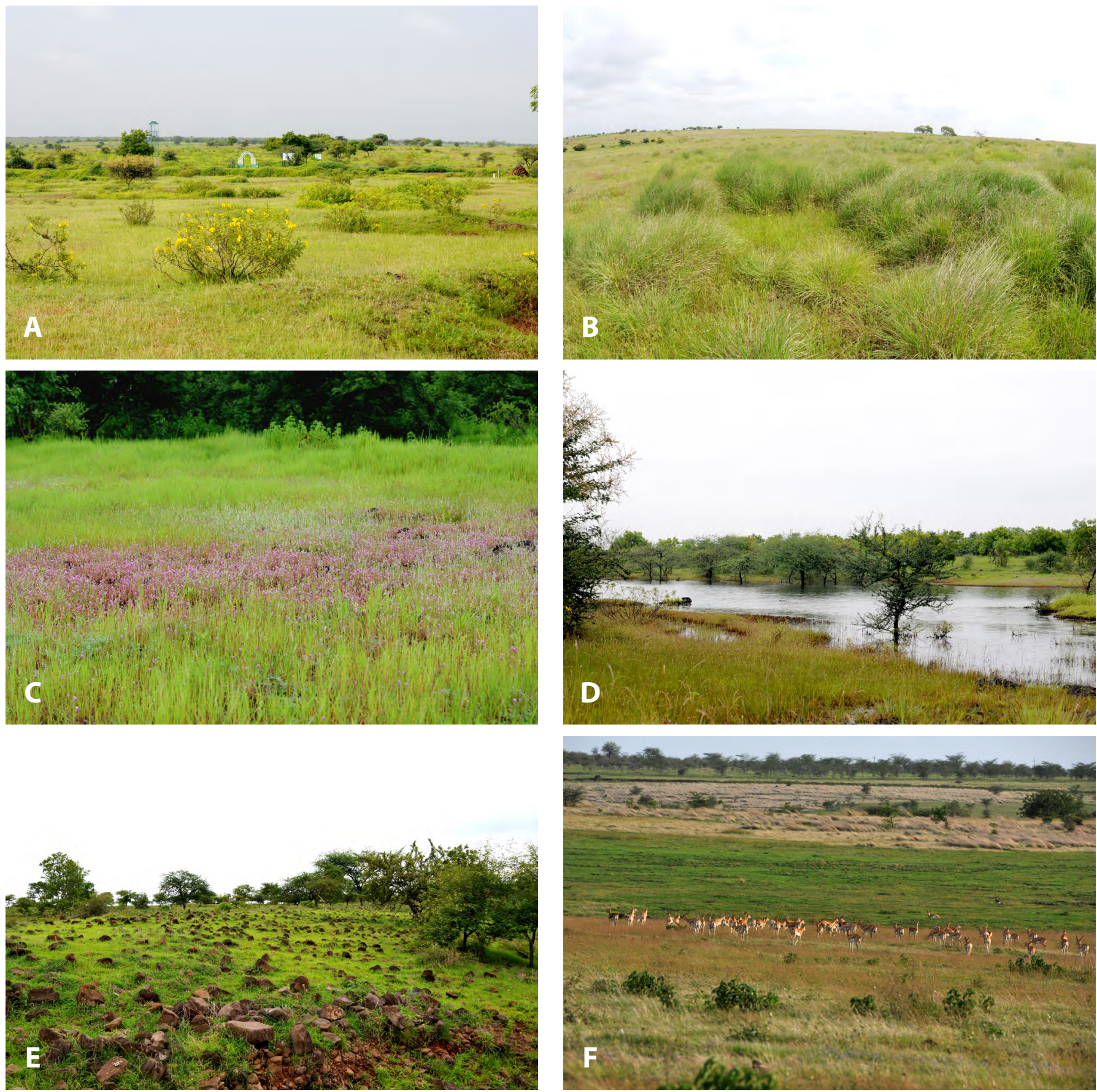

Figure 6. View of different habitats in Great Indian Bustard Wildlife sanctuary. A: View of core area at Nannaj. B: Clumps of grass dominated by Cymbopogon spp. C: Population of Cyanotis fasciculata amidst grasses. D: Seasonal lake inside the sanctuary showing scrub vegetation. E: View of rocky plateau inside the sanctuary. F: Herd of Black-buck population during monsoon season. 

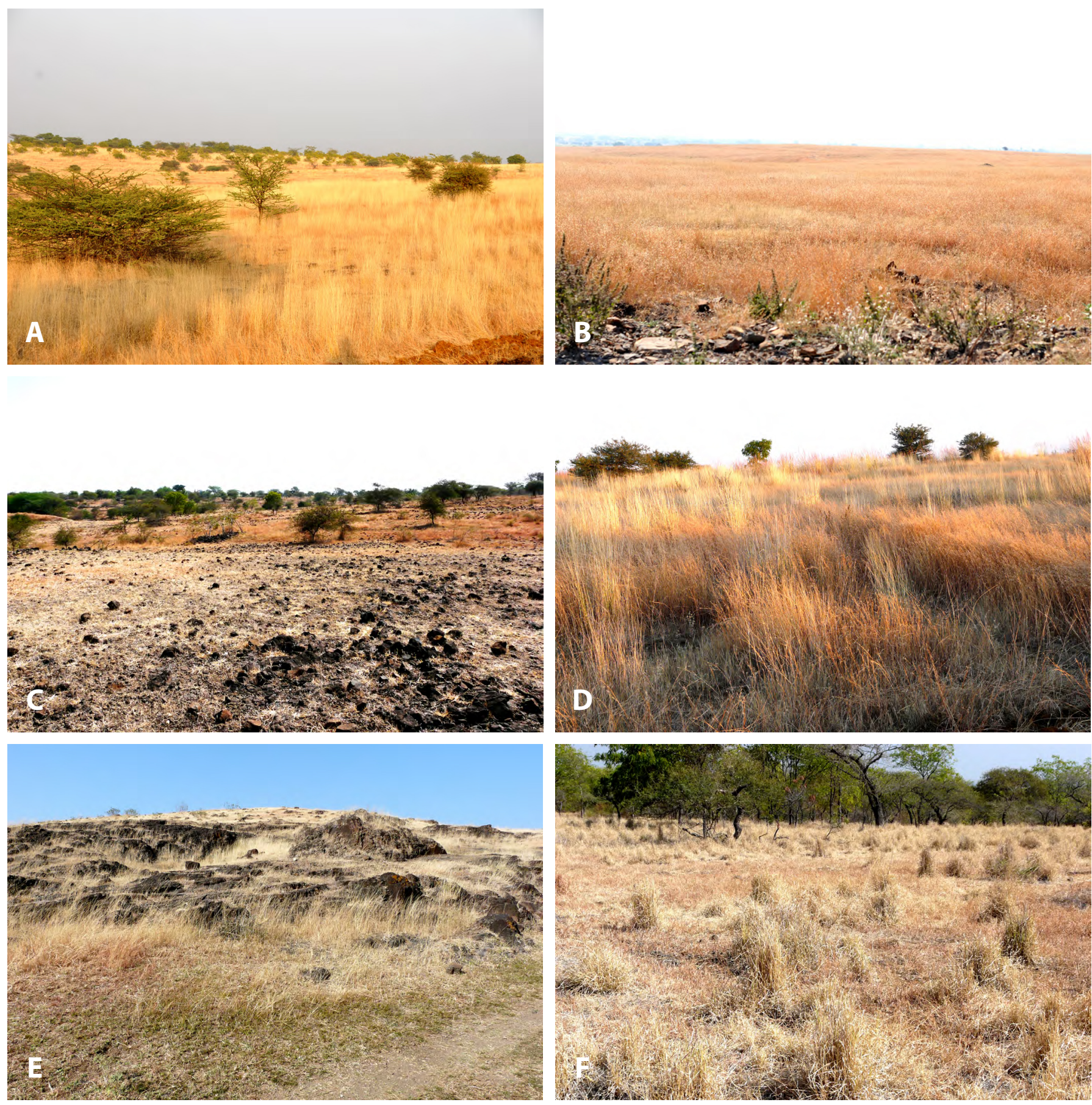

Figure 7. Different views of the sanctuary during dry season. A: View of grassland at Mardi. B: View at Karmala. C: View at Chapadgoan. D: View at Nannaj. E: View at Korti. F: View at Rehkuri. 

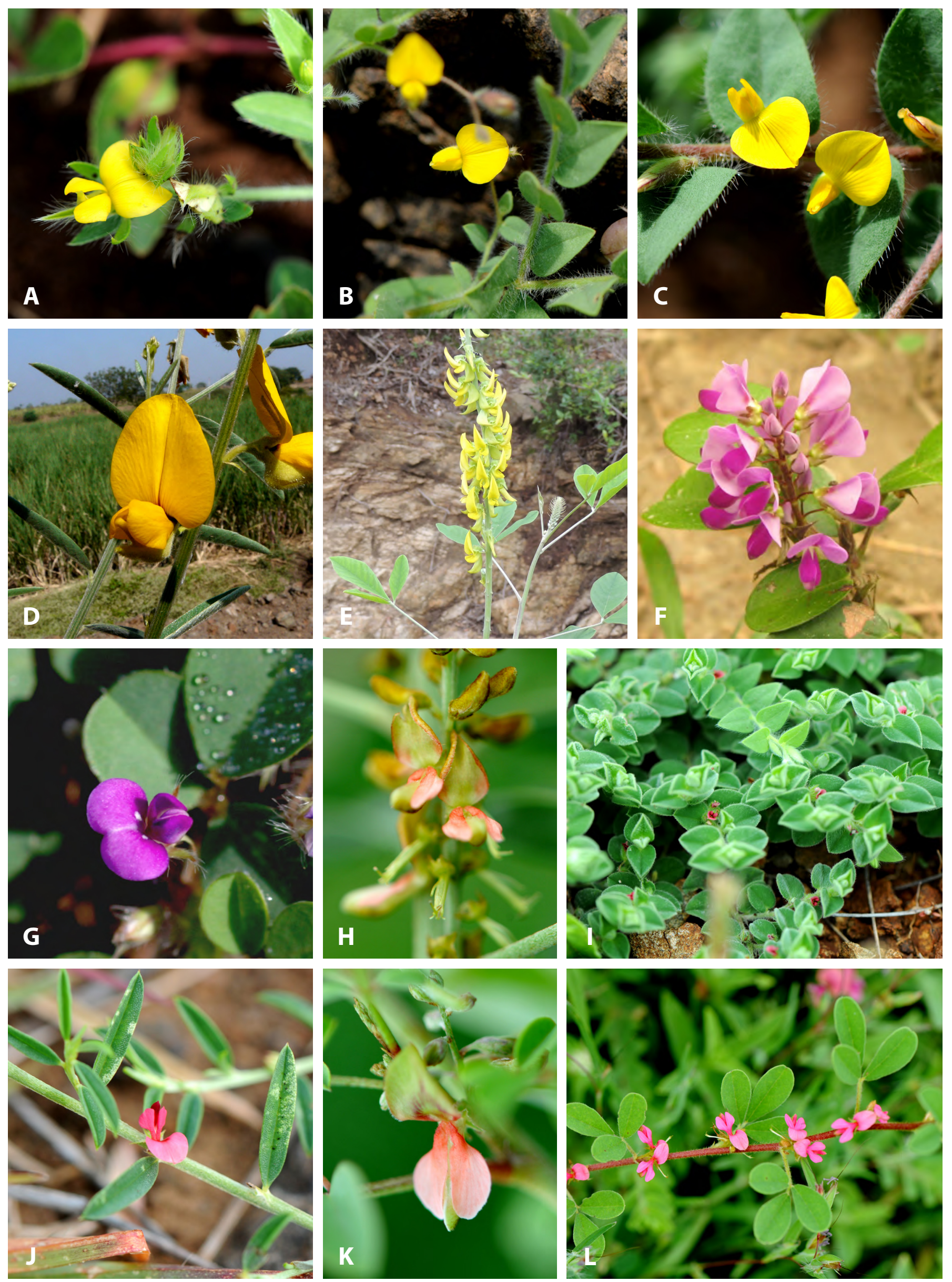

Figure 8. Legumes of the sanctuary. A: Crotalaria calycina. B: C. filipes. C: C. hebecarpa. D: C. juncea. E: C. pallida. F: Desmodium heterocarpon. G: $D$. triflorum. H: Indigofera coerulea. I: I. cordifolia. J: I. linifolia. K: I. tinctoria. L: I. trifoliata. 

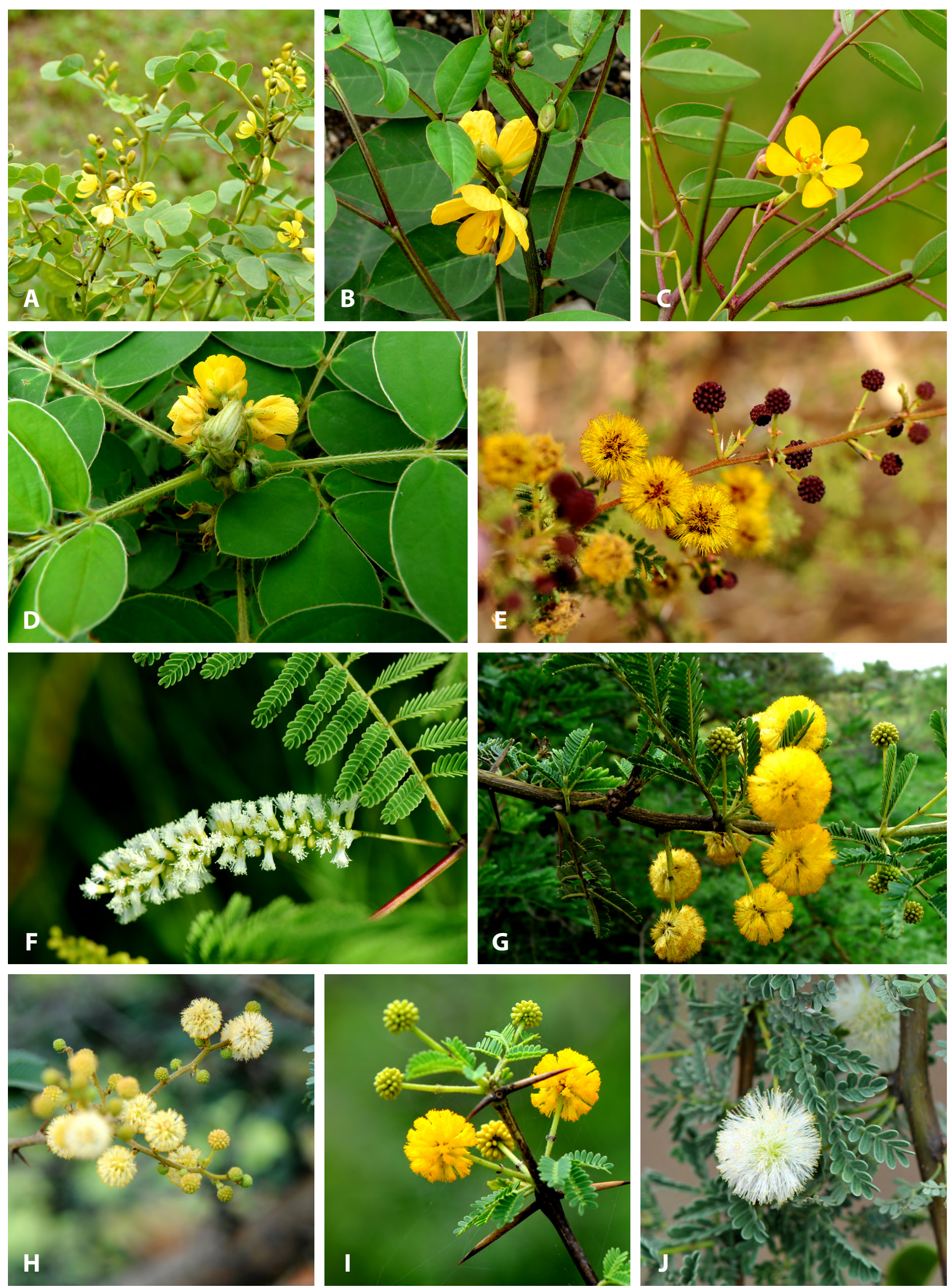

Figure 9. Senna and Acacia species found in the sanctuary. A: Senna italica. B: S. occidentalis. C: S. sophera. D: S. uniflora. E: Acacia campbelii. F: A. catechu. G: A. eburnea. H: A. leucophloea. I: A. nilotica. J: A. tomentosa. 

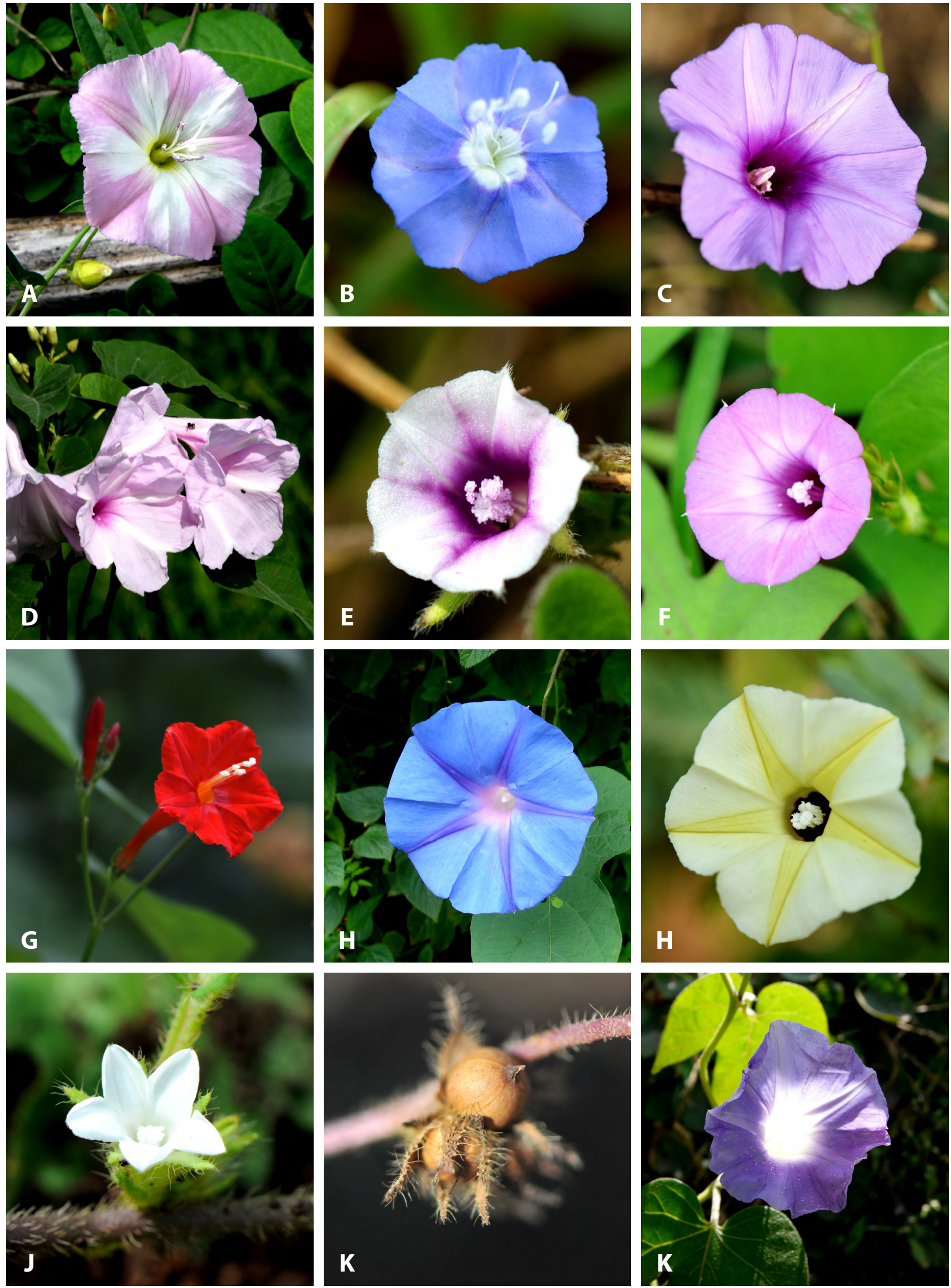

Figure 10. "Morning glories" recorded from the sanctuary. A: Convolvulus arvensis. B: Evolvulus alsinoides. C: Ipomoea cairica. D: I. carnea. E: I. eriocarpa. F: I. grandifolia. G: I. hederifolia. H: I. nil. I: I. obscura. J: I. sindica- in flower. K: I. sindica - in fruit. L: I. violacea. 

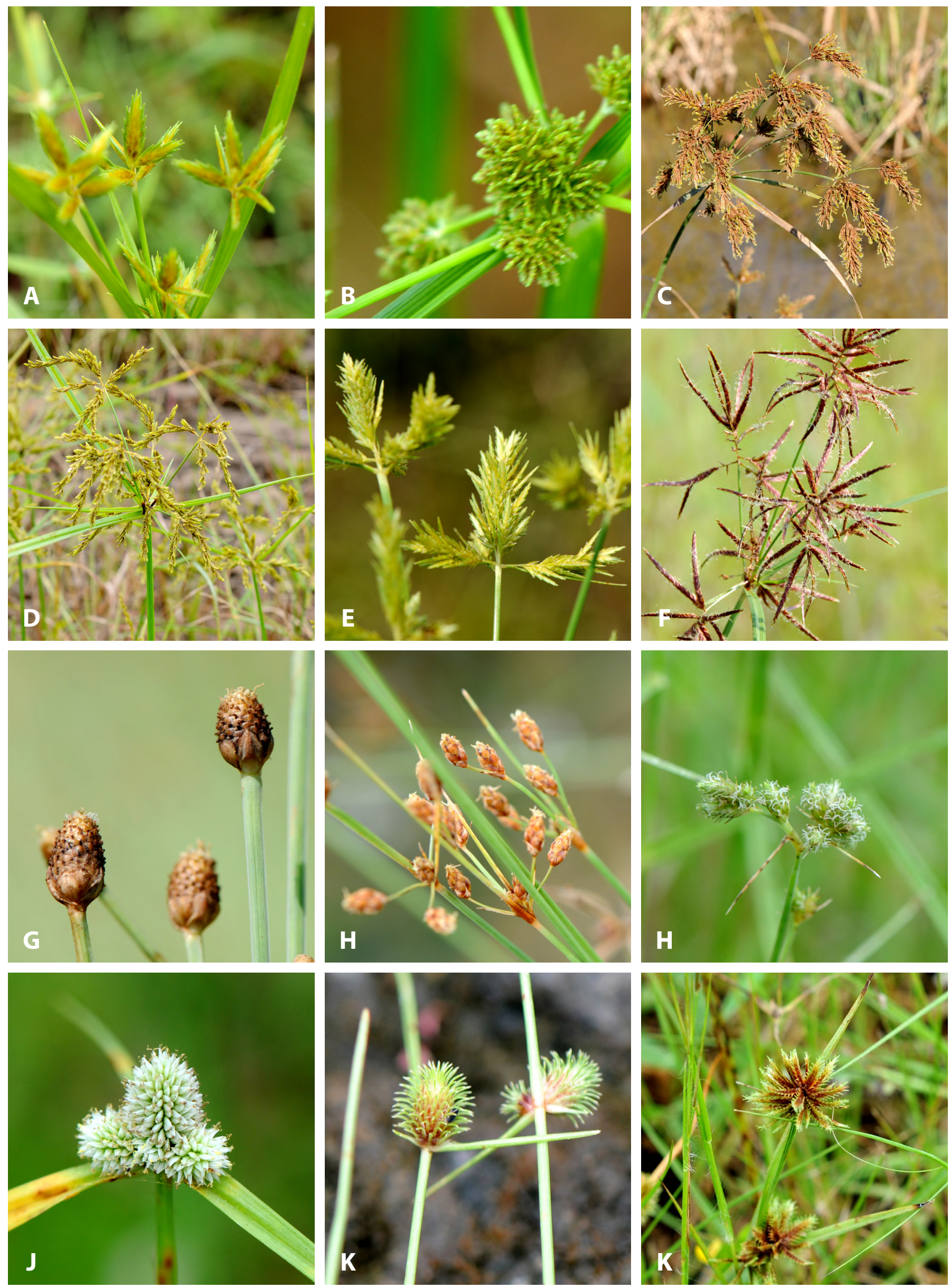

Figure 11. Sedges of the sanctuary. A: Cyperus compressus. B: C. difformis. C: C. digitatus. D: C. iria. E: C. nutans var. eleusinoides. F: C. rotundus. G: Eleocharis geniculata. H: Fimbristylis ferruginea. I: Fuirena cuspidata. J: Kyllinga brevifolia. K: Lipocarpha squarrosa. L: Mariscus squarrosus. 

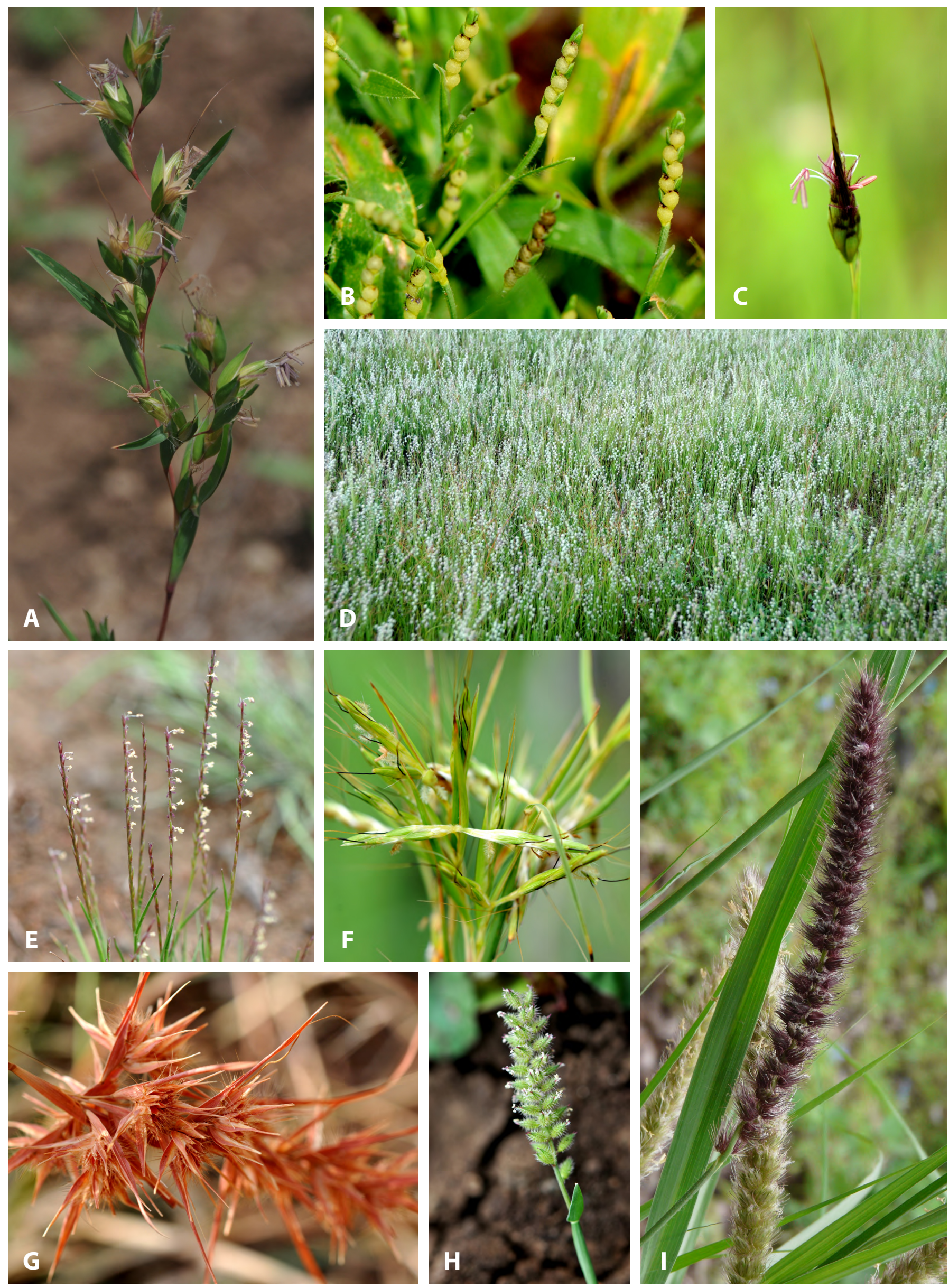

Figure 12. Grasses of the sanctuary. A: Apluda mutica. B: Hackelochloa granularis. C: Lophopogon tridentatus. D: Melanocenchris jacquemontii. E: Oropetium roxburghianum. F: Sehima ischaemoides.G: Themeda laxa. H: Tragus mongolorum. I: Pennisetum purpureum 